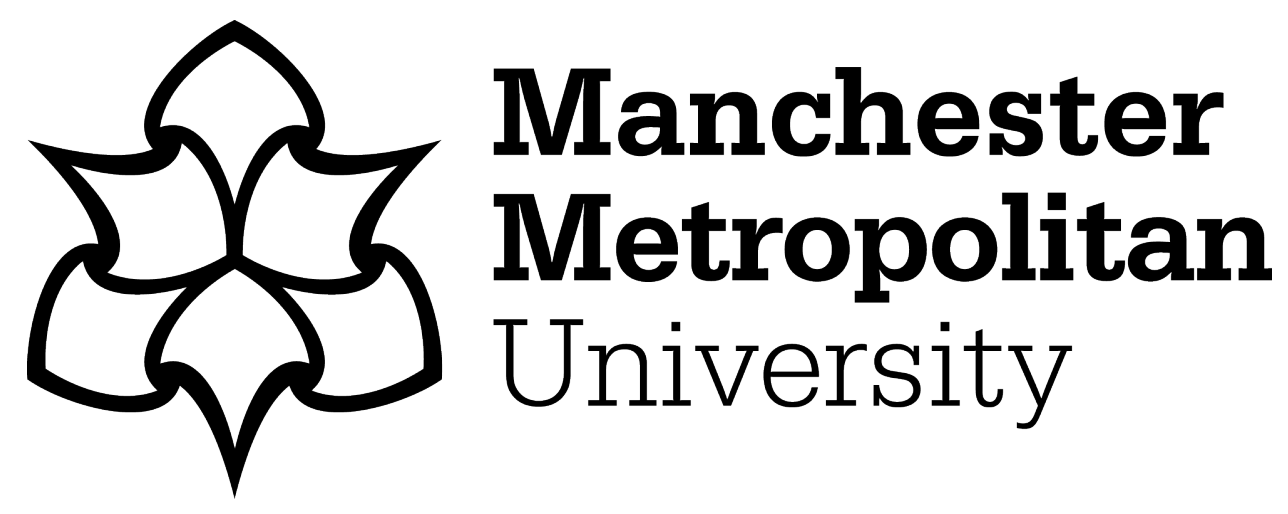

Cong, PW, Bai, W, Teng, B and Gou, Y (2018) Semi-analytical solution to the second-order wave loads on a vertical cylinder in bi-chromatic bi-directional waves. Ocean Engineering, 161. pp. 205-220. ISSN 0029-8018

Downloaded from: https://e-space.mmu.ac.uk/620468/

Version: Accepted Version

Publisher: Elsevier

DOI: https://doi.org/10.1016/j.oceaneng.2018.04.094

Usage rights: Creative Commons: Attribution-Noncommercial-No Derivative Works 4.0

Please cite the published version 


\title{
Semi-analytical solution to the second-order wave loads on a vertical cylinder in bi-chromatic bi-directional waves
}

\author{
Peiwen Cong ${ }^{\mathrm{a}} \mathrm{b}^{*}$, Wei Bai ${ }^{\mathrm{c}}$, Bin Teng ${ }^{\mathrm{a}}$ and Ying Gou ${ }^{\mathrm{a}}$ \\ ${ }^{a}$ State Key Laboratory of Coastal and Offshore Engineering, Dalian University of \\ Technology, Dalian 116024, China \\ ${ }^{\mathrm{b}}$ Department of Civil and Environmental Engineering, National University of \\ Singapore, 117576, Singapore \\ ${ }^{c}$ School of Computing, Mathematics and Digital Technology, Manchester \\ Metropolitan University, Chester Street, Manchester M1 5GD, UK
}

\begin{abstract}
\end{abstract}
A complete solution is presented for the second-order wave loads experienced by a uniform vertical cylinder in bi-chromatic bi-directional waves. The solution is obtained based on the introduction of an assisting radiation potential without explicitly evaluating the second-order diffraction potential. The semi-analytical formulation for calculating the wave loads is provided and an efficient numerical technique is developed to treat the oscillatory free-surface integral that appears in the force formulation. After validating the present solution by comparing with the predictions based on other methods, numerical studies are conducted for different combinations of incident wave frequencies and wave headings, and the influence of frequencies and headings of dual waves on the second-order wave loads is investigated. In addition, by expressing the second-order wave loads in a power expansion with respect to the wave frequency difference and wave heading difference which are both assumed to be small, approximations on the calculation of wave loads are developed. The accuracy of different approximations is assessed by comparing the approximate results with those based on the complete solution. 
30 Keywords: wave force; semi-analytical solution; bi-directional waves; bi-chromatic waves; second order

\section{Introduction}

In the offshore environment, the action of water waves is the primary source of external loads that need to be considered in the design of offshore structures. In the framework of potential flow theory, the perturbation procedure provides a powerful tool to investigate wave-body interaction problems, by which the linear, second-order and even higher-order models have been derived and implemented successfully in the past. The interaction of waves with arbitrary three-dimensional bodies can be in principle simulated numerically by the boundary element or finite element method. Although advances have been achieved in the numerical techniques associated with these approaches, the intensive computation still requires considerable amounts of CPU time and consumes large amounts of memory. In this regard, some researchers have considered idealized geometries, such as a circular cylinder (either bottom-mounted or truncated) and cylinder array, to approximate ocean structures and employed the analytical and semi-analytical approach to evaluate the hydrodynamic loads.

So far, the solution of the first-order wave-body interaction problem has progressed with great success and analytical solutions for fundamental geometric structures have been explored by many researchers. Examples include Garrett (1971), Yeung (1981), Kagemoto and Yue (1986), Linton and Evans (1990), Y1lmaz and Incecik (1998), Wu et al. (2006), Siddorn and Eatock Taylor (2008), Zheng and Zhang (2016), Liu et al. (2016) and Göteman (2017). In an irregular sea, consisting of a superposition of regular wave components, second-order high- and low-frequency hydrodynamic forces arise at the sum and difference frequencies of the constituent linear waves. These non-linear wave loads can play an important role in exciting some important phenomena, such as slow drift and springing (Petrauskas and Liu, 1988; Eatock Taylor and Kernot, 1999). Therefore the second-order interaction between waves and structures has also attracted 
continuous attention from the researchers. For example, by utilizing the so-called indirect method (Lighthill, 1979; Molin, 1979) which is based on the introduction of an assisting radiation potential to calculate the second-order wave loads without explicitly evaluating the second-order diffraction potential, semi-analytical formulations for the second-order wave force applied on fundamental geometric structures have been presented by Eatock Taylor and Hung (1987), Abul-Azm and Williams (1988, 1989), Ghalayini and Williams (1991), and Moubayed and Williams (1995). On the other hand, more direct methods including the second-order potential itself were adopted in Kim and Yue (1990), Chau and Eatock Taylor (1992), Huang and Eatock Taylor (1996), Teng and Kato (1999), Malenica et al. (1999).

The previous studies on the second-order wave diffraction primarily concern the action of unidirectional waves. However, to ensure a reliable design of offshore structures, it is of great demand to better understand the characteristics of the secondorder wave-body interaction with respect to the wave directional spreading. If all the wave components in directional seas are assumed to be independent, the wave forces can be obtained from the superposition of directional component waves. However, Eatock Taylor et al. (1988) indicated that this kind of superposition may not yield reliable results if the second-order effects are included. Therefore, some researchers developed the design methods which include both the second-order effects and wave directionality to investigate the properties of the second-order hydrodynamic loads induced by unidirectional waves. Kim (1992) developed a numerical model to predict the second-order difference-frequency wave forces on a large three-dimensional body in multi-directional waves based on the boundary integral equation method; Kim (1993) extended the asymptotic solution of the second-harmonic potential originally developed by Newman (1990) for the unidirectional wave to the multi-directional wave, and approximately evaluated the second-harmonic vertical forces on arrays of deep-draft vertical circular cylinders in monochromatic bi-directional waves. Based on the force formula on slender bodies proposed by Rainey (1989), Kim and Chen (1994) developed a slender-body approximation for the second-order difference-frequency wave force. 
Vazquez (1995) combined the boundary element method and indirect method to develop a solution for the second-order hydrodynamic loads on ocean structures in bichromatic bi-directional waves. Renaud et al. (2008) extended the middle-field formulation to the cases of bi-directional incident waves and performed calculation of wave drift loads and low-frequency loads on a LNG carrier.

In this study, the second-order interactions of plane bi-chromatic bi-directional incident waves with a vertical cylinder are considered, which is not well understood so far, but closely relevant to the design of marine structures in realistic ocean conditions. A complete solution for the second-order wave loads is developed based on the indirect method. The total second-order wave loads contain different constituent components which are related to the first-order interaction, the second-order incident potential and the second-order diffraction potential respectively. By utilizing Green's second identity, the force component associated with the second-order diffraction potential is expressed in terms of the free-surface and the body-surface integrals involving the first-order quantities and an assisting radiation potential. Evaluation of the oscillatory free-surface integral appears in the force formulation is the main difficulty in the calculation. Considering the effects of wave directionality, Kim (1992) developed a semi-analytical solution for the second-order difference-frequency wave force on a vertical cylinder, in which the local-wave-free integral method was used to predict the free-surface integral. In this study, another robust algorithm, which was proposed by Chau (1989) to determine successfully the second-order diffraction potential, is employed to calculate the infinite free-surface integral. By applying the integration by parts and the Bessel differential equation, the integral over the far-field free surface is transformed to a new expression without any derivative term, which is convenient for the numerical implementation. The solution is developed for both the difference-frequency and sumfrequency problems in this study. Moreover, efforts have been devoted to make the simplification on the calculation of second-order hydrodynamic loads, which is another contribution of the present study. By means of a power expansion with respect to the wave frequency difference and wave heading difference, both of which are assumed to 
be small, efficient approximations on the calculation of second-order hydrodynamic loads are eventually obtained.

\section{Governing equation and boundary conditions}

We consider the second-order interactions of plane bi-chromatic bi-directional incident waves with a bottom-mounted vertical circular cylinder. The cylinder is of radius $a$ and situated in the water of constant depth $d$. A cylindrical polar coordinate system $(r, \theta, z)$ is employed, with the origin on the undisturbed water surface and $z$ pointing upwards. The axis of the cylinder coincides with the $z$-axis. The definition of the coordinate system is given in Fig. 1. Assuming the flow to be irrotational, the fluid velocity at time $t$ is defined by the gradient of a velocity potential satisfying Laplace's equation. For unbroken waves, the wave steepness $\varepsilon$ is usually a small parameter and the velocity potential $\Phi$ can then be written as a perturbation series with respect to $\varepsilon$.

$$
\Phi=\varepsilon \Phi^{(1)}+\varepsilon^{2} \Phi^{(2)}+O\left(\varepsilon^{3}\right) .
$$

In Eq. (1), the superscripts (1) and (2) represent the first-order and second-order quantities, respectively.

With the presence of two plane incident waves of frequencies $\omega_{1}$ and $\omega_{2}$, the total first-order velocity potential can be expressed in the form:

$$
\Phi^{(1)}(\mathbf{x}, t)=\operatorname{Re}\left[\sum_{j=1}^{2} \phi_{j}^{(1)}(\mathbf{x}) e^{-i \omega_{j} t}\right],
$$

where, 'Re' indicates that the real part of the expression; $\phi_{j}^{(1)}$ represents the first-order velocity potential of the $j$ th wave component. The second-order potential can be written as a superposition of the sum- and difference-frequency terms:

$$
\Phi^{(2)}(\mathbf{x}, t)=\operatorname{Re}\left\{\sum_{j=1}^{2} \sum_{l=1}^{2}\left[\phi_{j l}^{(2)+}(\mathbf{x}) e^{-i\left(\omega_{j}+\omega_{l}\right) t}+\phi_{j l}^{(2)-}(\mathbf{x}) e^{-i\left(\omega_{j}-\omega_{l}\right) t}\right]\right\} .
$$

The second-order sum- and difference-frequency potentials in Eq. (3), $\phi_{j l}^{(2)+}$ and $\phi_{j l}^{(2)-}$, can be solved independently after separating the forcing terms and the boundary value problems accordingly. 
According to the concept of splitting the velocity potential into a certain number of components to properly satisfy the associated boundary conditions, $\phi_{j}^{(1)}$ is further decomposed into two parts:

$$
\phi_{j}^{(1)}=\phi_{j, I}^{(1)}+\phi_{j, D}^{(1)}, \quad(j=1,2),
$$

where $\phi_{j, I}^{(1)}$ and $\phi_{j, D}^{(1)}$ represent the first-order incident and diffraction potentials, respectively. The first-order incident and diffraction potentials can be expressed in a Fourier series in terms of the polar angle $\theta$ (Chau and Eatock Taylor 1992):

$$
\begin{aligned}
& \phi_{j, I}^{(1)}(r, \theta, z)=\sum_{m=-\infty}^{\infty} \varphi_{j, I, m}^{(1)}(r, z) e^{i m \theta} ; \\
& \phi_{j, D}^{(1)}(r, \theta, z)=\sum_{m=-\infty}^{\infty} \varphi_{j, D, m}^{(1)}(r, z) e^{i m \theta},
\end{aligned}
$$

where

$$
\begin{gathered}
\varphi_{j, I, m}^{(1)}(r, z)=-\frac{i A_{j} g}{\omega_{j}} \frac{\cosh \mu_{j}(z+d)}{\cosh \mu_{j} d} i^{m} e^{-i m \beta_{j}} J_{m}\left(\mu_{j} r\right) \\
\varphi_{j, D, m}^{(1)}(r, z)=\frac{i A_{j} g}{\omega_{j}} \frac{\cosh \mu_{j}(z+d)}{\cosh \mu_{j} d} \frac{J_{m}^{\prime}\left(\mu_{j} a\right)}{H_{m}^{\prime}\left(\mu_{j} a\right)} i^{m} e^{-i m \beta_{j}} H_{m}\left(\mu_{j} r\right) .
\end{gathered}
$$

In Eq. (6), $J_{m}(x)$ is the Bessel function of order $m ; H_{m}(x)$ is the first kind Hankel function of order $m ; g$ is the gravitational acceleration; $\mu_{j}(j=1,2)$ is the wave number satisfying the dispersion relation $\omega_{j}^{2}=g \mu_{j} \tanh \mu_{j} d ; A_{j}$ and $\beta_{j}(j=1,2)$ are the amplitude and heading of the $j$ th incident wave component, respectively. After expanding $\phi_{j}^{(1)}$ into the Fourier series in the circumferential coordinate $\theta$, we can obtain that

$$
\phi_{j}^{(1)}(r, \theta, z)=\sum_{m=-\infty}^{\infty} \varphi_{j, m}^{(1)}(r, z) e^{i m \theta}
$$

where

$$
\varphi_{j, m}^{(1)}(r, z)=\varphi_{j, I, m}^{(1)}(r, z)+\varphi_{j, D, m}^{(1)}(r, z)
$$

Similarly, the second-order velocity potential can be decomposed into the incident the diffraction potentials: 


$$
\phi_{j l}^{(2) \pm}=\phi_{j l, I}^{(2) \pm}+\phi_{j l, D}^{(2) \pm}, \quad(j, l=1,2) .
$$

In the presence of bi-chromatic bi-directional incident waves, a symmetric form of the second-order sum- and difference-frequency incident potentials were given by Kim (1992, 1993):

$$
\phi_{j l, I}^{(2) \pm}(r, \theta, z)=\sum_{m=-\infty}^{\infty} \varphi_{j l, I, m}^{(2) \pm}(r, z) e^{-i m \theta}
$$

in which

$$
\begin{gathered}
\varphi_{j l, I, m}^{(2)+}(r, z)=\frac{1}{2}\left(\gamma_{j l}^{+}+\gamma_{l j}^{+}\right) \frac{\cosh \mu_{j l}^{+}(z+d)}{\cosh \mu_{j l}^{+} d} i^{m} e^{-i m \beta_{j l}^{+}} J_{m}\left(\mu_{j l}^{+} r\right) ; \\
\varphi_{j l, I, m}^{(2)-}(r, z)=\frac{1}{2}\left(\gamma_{j l}^{-}+\gamma_{l j}^{-*}\right) \frac{\cosh \mu_{j l}^{-}(z+d)}{\cosh \mu_{j l}^{-} d} i^{m} e^{-i m \beta_{j l}^{-}} J_{m}\left(\mu_{j l}^{-} r\right),
\end{gathered}
$$

where

$$
\begin{gathered}
\gamma_{j l}^{+}=-\frac{i A_{j} A_{l} g^{2}}{2 \omega_{j}} \frac{\mu_{j}^{2}\left(1-\tanh ^{2} \mu_{j} d\right)+2 \mu_{j} \mu_{l}\left[\cos \left(\beta_{j}-\beta_{l}\right)-\tanh \mu_{j} d \tanh \mu_{l} d\right]}{\left(\omega_{j}+\omega_{l}\right)^{2}-g \mu_{j l}^{+} \tanh \mu_{j l}^{+} d} \\
\gamma_{j l}^{-}=-\frac{i A_{j} A_{l}^{*} g^{2}}{2 \omega_{j}} \frac{\mu_{j}^{2}\left(1-\tanh ^{2} \mu_{j} d\right)-2 \mu_{j} \mu_{l}\left[\cos \left(\beta_{j}-\beta_{l}\right)+\tanh \mu_{j} d \tanh \mu_{l} d\right]}{\left(\omega_{j}-\omega_{l}\right)^{2}-g \mu_{j l}^{-} \tanh \mu_{j l}^{-} d} .
\end{gathered}
$$

In the above equations, an asterisk represents the complex conjugate, and $\mu_{j l}^{ \pm}$and $\beta_{j l}^{ \pm}$ are defined respectively by

$$
\mu_{j l}^{ \pm}=\sqrt{\mu_{j}^{2}+\mu_{l}^{2} \pm 2 \mu_{j} \mu_{l} \cos \left(\beta_{j}-\beta_{l}\right)}
$$

and

$$
\beta_{j l}^{ \pm}=\tan ^{-1}\left(\frac{\mu_{j} \sin \beta_{j} \pm \mu_{l} \sin \beta_{l}}{\mu_{j} \cos \beta_{j} \pm \mu_{l} \cos \beta_{l}}\right)
$$

As the amplitude of the free surface displacement is small, the boundary conditions satisfied on the instantaneous free surface can be expanded into the Taylor series about the still water surface. The sum- and difference-frequency components of the secondorder diffraction potential, $\phi_{j l, D}^{(2) \pm}$, satisfy the following boundary value problems:

$$
\nabla^{2} \phi_{j l, D}^{(2) \pm}=0
$$




$$
\frac{\partial \phi_{j l, D}^{(2) \pm}}{\partial r}=-\frac{\partial \phi_{j l, I}^{(2) \pm}}{\partial r}, \quad \text { on } r=a
$$

$$
\frac{\partial \phi_{j l, D}^{(2) \pm}}{\partial z}=\frac{\left(\omega_{j} \pm \omega_{l}\right)^{2}}{g} \phi_{j l, D}^{(2) \pm}+\frac{1}{g} q_{j l, D}^{(2) \pm}(r, \theta), \quad \text { on } z=0
$$

$$
\frac{\partial \phi_{j l, D}^{(2) \pm}}{\partial z}=0, \quad \text { on } z=-d \text {. }
$$

189 In Eq. (15c), $q_{j l, D}^{(2) \pm}$ are the non-homogeneous sum- and difference-frequency free190 surface forcing terms and can be expressed as follows:

$$
\begin{gathered}
q_{j l, D}^{(2)+}=-\frac{1}{4} i\left(\omega_{j} \alpha_{l j}+\omega_{l} \alpha_{j l}\right)\left(\phi_{j}^{(1)} \phi_{l}^{(1)}-\phi_{j, I}^{(1)} \phi_{l, I}^{(1)}\right) \\
+\frac{1}{2} i\left(\omega_{j}+\omega_{l}\right)\left(\frac{\partial \phi_{j}^{(1)}}{\partial r} \frac{\partial \phi_{l}^{(1)}}{\partial r}+\frac{1}{r^{2}} \frac{\partial \phi_{j}^{(1)}}{\partial \theta} \frac{\partial \phi_{l}^{(1)}}{\partial \theta}-\frac{\partial \phi_{j, I}^{(1)}}{\partial r} \frac{\partial \phi_{l, I}^{(1)}}{\partial r}-\frac{1}{r^{2}} \frac{\partial \phi_{j, I}^{(1)}}{\partial \theta} \frac{\partial \phi_{l, I}^{(1)}}{\partial \theta}\right) \\
q_{j l, D}^{(2)-}=-\frac{1}{4} i\left(\omega_{j} \alpha_{l j}-\omega_{l} \alpha_{j l}\right)\left(\phi_{j}^{(1)} \phi_{l}^{(1) *}-\phi_{j, I}^{(1)} \phi_{l, I}^{(1) *}\right) \\
+\frac{1}{2} i\left(\omega_{j}-\omega_{l}\right)\left(\frac{\partial \phi_{j}^{(1)}}{\partial r} \frac{\partial \phi_{l}^{(1) *}}{\partial r}+\frac{1}{r^{2}} \frac{\partial \phi_{j}^{(1)}}{\partial \theta} \frac{\partial \phi_{l}^{(1) *}}{\partial \theta}-\frac{\partial \phi_{j, I}^{(1)}}{\partial r} \frac{\partial \phi_{l, I}^{(1) *}}{\partial r}-\frac{1}{r^{2}} \frac{\partial \phi_{j, I}^{(1)}}{\partial \theta} \frac{\partial \phi_{l, I}^{(1) *}}{\partial \theta}\right)
\end{gathered}
$$

193 where

$$
\alpha_{j l}=\mu_{j}^{2}\left(1-\tanh ^{2} \mu_{j} d\right)-2 \mu_{j} \mu_{l} \tanh \mu_{j} d \tanh \mu_{l} d
$$

195

At the far field, an appropriate radiation condition for the second-order doublefrequency potential was first obtained by Molin (1979). Then, the analysis was extended by Kim and Yue (1990) to the bi-chromatic problem. After performing a local far-field asymptotic analysis, Kim and Yue (1990) suggested that the second-order diffraction potentials at the sum and difference frequencies decay at a rate of $1 / \sqrt{r}$ as $r$ extends to infinity, and a weak radiation condition at the far field can then be guaranteed.

\section{Calculation of the Second-order Wave Force}

Similar to the velocity potential, the wave force can be expanded in a perturbation series in terms of the wave steepness parameter $\varepsilon$ as:

$$
\mathbf{F}=\mathbf{F}^{(0)}+\varepsilon \mathbf{F}^{(1)}+\varepsilon^{2} \mathbf{F}^{(2)}+O\left(\varepsilon^{3}\right) .
$$


In the presence of bi-chromatic incident waves, the second-order hydrodynamic loads, $\mathbf{F}^{(2)}$, contain the sum- and difference-frequency components. When a periodic wave motion is assumed, $\mathbf{F}^{(2)}$ can be expressed in the time spatial decomposed form as:

$$
\mathbf{F}^{(2)}=\sum_{j=1}^{2} \sum_{l=1}^{2}\left[\mathbf{f}_{j l}^{(2)+} e^{-i\left(\omega_{j}+\omega_{l}\right) t}+\mathbf{f}_{j l}^{(2)-} e^{-i\left(\omega_{j}-\omega_{l}\right) t}\right],
$$

where $\mathbf{f}_{j l}^{(2) \pm}$ are the second-order sum- and difference-frequency wave exciting forces. Ogilvie (1983) derived the expressions of the second-order wave loads applied on a three-dimensional body in the presence of monochromatic incident waves. These expressions have been extended to the cases of bi-directional bi-chromatic incident waves in Kim (1992) and Vazquez (1995). The second-order sum- and differencefrequency wave loads exerted on a vertical cylinder in bi-directional bi-chromatic incident waves can be determined according to:

$$
\mathbf{f}_{j l}^{(2)+}=i\left(\omega_{j}+\omega_{l}\right) \rho \iint_{S_{b}} \phi_{j l}^{(2)+} \mathbf{n} d s-\frac{\rho}{4} \iint_{S_{b}} \nabla \phi_{j}^{(1)} \cdot \nabla \phi_{l}^{(1)} \mathbf{n} d s-\frac{\rho}{4} \frac{\omega_{j} \omega_{l}}{g} \underset{\dot{C}_{w}}{\circ}
$$

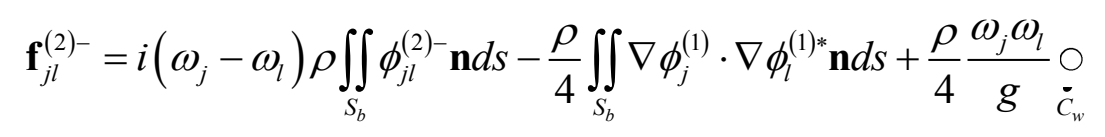

$C_{w}$ and $S_{b}$ represent the waterline and the immersed body surface in calm water, respectively, and $\mathbf{n}$ is the normal vector on $S_{b}$ pointing out of the fluid domain.

It can be noted that there are three terms on the right-hand side of Eq. (20). The integrand of the first and second terms come directly from the terms in the Bernoulli equation. Meanwhile, the third term arises from the operation in correcting the integral on instantaneous wetted body surface to that on $S_{b}$. In addition, the total second-order wave loads in general contain two separated contributions. One is from the first term on the right-hand side of Eq. (20), which is due to the second-order potential itself. The other one is from the remaining two terms on the right-hand side of Eq. (20), which is due to the quadratic products of first-order potentials. Hereinafter, they are denoted by $\mathbf{f}_{j l, p}^{(2) \pm}$ and $\mathbf{f}_{j l, q}^{(2) \pm}:$

$$
\mathbf{f}_{j l}^{(2) \pm}=\mathbf{f}_{j l, p}^{(2) \pm}+\mathbf{f}_{j l, q}^{(2) \pm} .
$$


and

$$
\mathbf{f}_{j l, p}^{(2) \pm}=i\left(\omega_{j} \pm \omega_{l}\right) \rho \iint_{S_{b}} \phi_{j l}^{(2) \pm} \mathbf{n} d s=i\left(\omega_{j} \pm \omega_{l}\right) \rho \iint_{S_{b}}\left(\phi_{j l, I}^{(2) \pm}+\phi_{j l, D}^{(2) \pm}\right) \mathbf{n} d s
$$

7

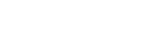

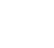

in which 


$$
G_{j, m}=\frac{2 A_{j} g}{\omega_{j} \pi \mu_{j} a} \frac{i^{m} e^{-i m \beta_{j}}}{H_{m}^{\prime}\left(\mu_{j} a\right)}
$$

and

$$
\Omega_{j l}^{ \pm}=\frac{1}{2 \cosh \mu_{j} d \cosh \mu_{l} d}\left[\frac{\sinh \left(\mu_{j}+\mu_{l}\right) d}{\mu_{j}+\mu_{l}} \pm \frac{\sinh \left(\mu_{j}-\mu_{l}\right) d}{\mu_{j}-\mu_{l}}\right] .
$$

In Eq. (24), the upper elements in the bracketed pair refer to the force in the $x$-direction and the lower elements in the $y$-direction. In Eq. (25), the prime appearing in the superscript denotes the differentiation with respect to the argument.

The contribution due to the second-order incident potential, $\mathbf{f}_{j l, I}^{(2) \pm}$, can be evaluated directly. After integration in $\theta, f_{j l, I, x}^{(2) \pm}$ and $f_{j l, I, y}^{(2) \pm}$ can be expressed as

$$
\left\{\begin{array}{l}
f_{j l, I, x}^{(2) \pm} \\
f_{j l, I, y}^{(2) \pm}
\end{array}\right\}=-i\left(\omega_{j} \pm \omega_{l}\right) \rho \pi a\left\{\begin{array}{l}
1 \\
i
\end{array}\right\}\left(G_{j l, 1}^{ \pm}\left\{\begin{array}{l}
+ \\
-
\end{array}\right\} G_{j l,-1}^{ \pm}\right) \frac{\tanh \mu_{j l}^{ \pm} d}{\mu_{j l}^{ \pm}}
$$

where

$$
\begin{aligned}
& G_{j l, m}^{+}=\frac{1}{2}\left(\gamma_{j l}^{+}+\gamma_{l j}^{+}\right) i^{m} e^{-i m \beta_{j l}^{+}} J_{m}\left(\mu_{j l}^{+} a\right) ; \\
& G_{j l, m}^{-}=\frac{1}{2}\left(\gamma_{j l}^{-}+\gamma_{l j}^{-*}\right) i^{m} e^{-i m \beta_{j l}^{-}} J_{m}\left(\mu_{j l}^{-} a\right) .
\end{aligned}
$$

The computation of $\mathbf{f}_{j l, d}^{(2) \pm}$ can be accomplished by utilizing a method developed by Lighthill (1979) for monochromatic waves in infinite water depths, which was adapted for finite water depths by Molin (1979) and later extended by several researchers. The method does not involve the explicit calculation of the second-order potential. Instead, through the application of Green's second identity, the loading components due to this potential can be expressed in terms of the free-surface and body-surface integrals involving the first-order quantities and an assisting radiation potential. For the calculation of the wave loads in the $x$-direction ( $y$-direction), the radiation potentials due to the forced harmonic motion of the vertical cylinder in the $x$-direction ( $y$-direction) at the sum frequency, $\omega_{j}+\omega_{l}$, or difference frequency, $\omega_{j}-\omega_{l}$, of interest are employed as the assisting potentials. In this study, $\Psi_{j l, x}^{ \pm}$and $\Psi_{j l, y}^{ \pm}$are defined as the assisting radiation potential and they are involved in the calculation of the wave loads in the $x$ - and $y$-directions respectively. $\Psi_{j l, x}^{ \pm}$and $\Psi_{j l, y}^{ \pm}$satisfy a homogeneous 
boundary condition on the free surface, while on the cylinder surface the following conditions hold:

$$
\begin{aligned}
& \frac{\partial \Psi_{j l, x}^{ \pm}}{\partial r}=\cos \theta, \quad \text { on } r=a ; \\
& \frac{\partial \Psi_{j l, y}^{ \pm}}{\partial r}=\sin \theta, \quad \text { on } r=a .
\end{aligned}
$$

The approach of separation of variables is applied in the fluid domain and yields the spatial potentials expressed by the orthogonal series for $\Psi_{j l, x}^{ \pm}$and $\Psi_{j l, y}^{ \pm}$. These expressions are developed to satisfy all the boundary conditions and Laplace's equation. $\Psi_{j l, x}^{ \pm}$and $\Psi_{j l, y}^{ \pm}$can then be expressed as (Rahman and Bhatta, 1993):

$$
\begin{aligned}
& \Psi_{j l, x}^{ \pm}(r, \theta, z)=\psi_{j l}^{ \pm}(r, z) \cos \theta ; \\
& \Psi_{j l, y}^{ \pm}(r, \theta, z)=\psi_{j l}^{ \pm}(r, z) \sin \theta,
\end{aligned}
$$

where

$$
\psi_{j l}^{ \pm}(r, z)=\frac{B_{j l, 0}^{ \pm} H_{1}\left(\kappa_{j l}^{ \pm} r\right)}{\kappa_{j l}^{ \pm} H_{1}^{\prime}\left(\kappa_{j l}^{ \pm} a\right)} \frac{\cosh \kappa_{j l}^{ \pm}(z+d)}{\cosh \kappa_{j l}^{ \pm} d}+\sum_{n=1}^{\infty} \frac{B_{j l, n}^{ \pm} K_{1}\left(\kappa_{j l, n}^{ \pm} r\right)}{\kappa_{j l, n}^{ \pm} K_{1}^{\prime}\left(\kappa_{j l, n}^{ \pm} a\right)} \frac{\cos \kappa_{j l, n}^{ \pm}(z+d)}{\cos \kappa_{j l, n}^{ \pm} d},
$$

in which

$$
B_{j l, n}^{ \pm}= \begin{cases}\frac{2 \sinh 2 \kappa_{j l}^{ \pm} d}{2 \kappa_{j l}^{ \pm} d+\sinh 2 \kappa_{j l}^{ \pm} d}, & n=0 ; \\ \frac{2 \sin 2 \kappa_{j l, n}^{ \pm} d}{2 \kappa_{j l, n}^{ \pm} d+\sin 2 \kappa_{j l, n}^{ \pm} d}, & n \geq 1,\end{cases}
$$

292

293

296

297

$\kappa_{j l}^{ \pm}$satisfies the dispersion relation $\left(\omega_{j} \pm \omega_{l}\right)^{2}=-g \kappa_{j l}^{ \pm} \tanh \left(\kappa_{j l}^{ \pm} d\right) ; \kappa_{j l, n}^{ \pm}(n \geq 1)$ are positive real roots of $\left(\omega_{j} \pm \omega_{l}\right)^{2}=-g \kappa_{j l, n}^{ \pm} \tan \left(\kappa_{j l, n}^{ \pm} d\right)$.

After getting the assisting radiation potential, we now use the Green's second identify on the surfaces enclosing the fluid domain:

$$
\begin{array}{r}
\iint_{S_{b} \cup S_{d} \cup S_{f} \cup S_{\infty}}\left(\phi_{j l, d}^{(2) \pm} \frac{\partial \Psi_{j l, x}^{ \pm}}{\partial n}-\Psi_{j l, x}^{ \pm} \frac{\partial \phi_{j l, d}^{(2) \pm}}{\partial n}\right) d s=0 ; \\
\iint_{S_{b} \cup S_{d} \cup S_{f} \cup S_{\infty}}\left(\phi_{j l, d}^{(2))} \frac{\partial \Psi_{j l, y}^{ \pm}}{\partial n}-\Psi_{j l, y}^{ \pm} \frac{\partial \phi_{j l, d}^{(2) \pm}}{\partial n}\right) d s=0,
\end{array}
$$


316 where

where

and

and

where $S_{\infty}$ is the surface of a circular cylinder containing the entire fluid domain; $S_{d}$ represents the sea bottom. The definition of the $S_{\infty}$ and $S_{d}$ can also be found in Fig. 1. By using the asymptotic expressions for Bessel functions (large arguments) and employing the theorem of the stationary phase, Molin (1979) has proved that the integral over $S_{\infty}$ oscillates towards zero when the radius of $S_{\infty}$ goes to infinity. Then imposing the seabed boundary condition and the structural boundary condition on $\phi_{j l, d}^{(2) \pm}$, $\Psi_{j l, x}^{ \pm}$and $\Psi_{j l, y}^{ \pm}$gives

$$
\left\{\begin{array}{l}
f_{j l, d, x}^{(2) \pm} \\
f_{j l, d, y}^{(2) \pm}
\end{array}\right\}=\left\{\begin{array}{c}
f_{j l, b, x}^{(2) \pm} \\
f_{j l, b, y}^{(2) \pm}
\end{array}\right\}+\left\{\begin{array}{c}
f_{j l, f, x}^{(2) \pm} \\
f_{j l, f, y}^{(2) \pm}
\end{array}\right\}
$$

$$
\left\{\begin{array}{c}
f_{j l, b, x}^{(2) \pm} \\
f_{j l, b, y}^{(2) \pm}
\end{array}\right\}=-i\left(\omega_{j} \pm \omega_{l}\right) \rho \iint_{S_{b}} \frac{\partial \phi_{j l, I}^{(2) \pm}}{\partial n}\left\{\begin{array}{c}
\Psi_{j l, x}^{ \pm} \\
\Psi_{j l, y}^{ \pm}
\end{array}\right\} d s,
$$

$$
\left\{\begin{array}{c}
f_{j l, f, x}^{(2) \pm} \\
f_{j l, f, y}^{(2) \pm}
\end{array}\right\}=\frac{i\left(\omega_{j} \pm \omega_{l}\right) \rho}{g} \iint_{S_{f}} q_{j l, D}^{(2) \pm}\left\{\begin{array}{l}
\Psi_{j l, x}^{ \pm} \\
\Psi_{j l, y}^{ \pm}
\end{array}\right\} d s
$$

The remaining task is to evaluate the body-surface and free-surface integrals in Eqs. (35) and (36). After substituting Eqs. (10) and (30) into the right-hand side of Eq. (35), the body-surface integrals can be reduced to the line integrals after integrating with respect to $\theta$ and using the orthogonal relationship. Thus, $f_{j l, b, x}^{(2) \pm}$ and $f_{j l, b, y}^{(2) \pm}$, can be written as

$$
\left\{\begin{array}{c}
f_{j l, b, x}^{(2) \pm} \\
f_{j l, b, y}^{(2) \pm}
\end{array}\right\}=i\left(\omega_{j} \pm \omega_{l}\right) \rho \pi a\left\{\begin{array}{l}
1 \\
i
\end{array}\right\}\left(\hat{G}_{j l, 1}^{ \pm}\left\{\begin{array}{l}
+ \\
-
\end{array}\right\} \hat{G}_{j l,-1}^{ \pm}\right) \sum_{n=0}^{\infty} \Pi_{j l, n}^{ \pm}
$$

$$
\begin{aligned}
& \hat{G}_{j l, m}^{+}=\frac{1}{2}\left(\gamma_{j l}^{+}+\gamma_{j l}^{+}\right) i^{m} e^{-i m \beta_{j l}^{+}} \mu_{j l}^{+} J_{m}^{\prime}\left(\mu_{j l}^{+} a\right) ; \\
& \hat{G}_{j l, m}^{-}=\frac{1}{2}\left(\gamma_{j l}^{-}+\gamma_{l j}^{-*}\right) i^{m} e^{-i m \beta_{j l}^{-}} \mu_{j l}^{-} J_{m}^{\prime}\left(\mu_{j l}^{-} a\right),
\end{aligned}
$$




$$
\Pi_{j l, n}^{ \pm}=\left\{\begin{array}{cl}
\frac{B_{0}^{ \pm} H_{1}\left(\kappa_{j l}^{ \pm} a\right)}{\kappa_{j l}^{ \pm} H_{1}^{\prime}\left(\kappa_{j l}^{ \pm} a\right)} \frac{\mu_{j l}^{+} \tanh \mu_{j l}^{+} d \cosh \kappa_{j l}^{ \pm} d-\kappa_{j l}^{ \pm} \sinh \kappa_{j l}^{ \pm} d}{\left(\mu_{j l}^{+}\right)^{2}-\left(\kappa_{j l}^{ \pm}\right)^{2}}, & n=0 ; \\
-\frac{B_{n}^{ \pm} K_{1}\left(\kappa_{j l, n}^{ \pm} a\right)}{\kappa_{j l, n}^{ \pm} K_{1}^{\prime}\left(\kappa_{j l, n}^{ \pm} a\right)} \frac{\mu_{j l}^{ \pm} \tanh \mu_{j l}^{ \pm} d \cos \kappa_{j l, n}^{ \pm} d-\kappa_{j l, n}^{ \pm} \sin \kappa_{j l, n}^{ \pm} d}{\left(\mu_{j l}^{+}\right)^{2}+\left(\kappa_{j l, n}^{ \pm}\right)^{2}}, & n \geq 1 .
\end{array}\right.
$$

The numerical evaluation of the free-surface integral appearing in Eq. (36)

322

$$
\begin{gathered}
u_{j l, k}^{+}=\sum_{m=-\infty}^{+\infty}\left\{\frac{1}{2} i\left(\omega_{l}+\omega_{j}\right)\left(\frac{\partial \varphi_{j, m}^{(1)}}{\partial r} \frac{\partial \varphi_{l,-m+k}^{(1)}}{\partial r}-\frac{\partial \varphi_{j, I, m}^{(1)}}{\partial r} \frac{\partial \varphi_{l, I,-m+k}^{(1)}}{\partial r}\right)\right. \\
\left.-\frac{1}{2} i\left[\frac{\omega_{l} \alpha_{j l}+\omega_{j} \alpha_{l j}}{2}-\left(\omega_{j}+\omega_{l}\right) \frac{m(m-k)}{r^{2}}\right]\left(\varphi_{j, m}^{(1)} \varphi_{l,-m+k}^{(1)}-\varphi_{j, I, m}^{(1)} \varphi_{l, I,-m+k}^{(1)}\right)\right\}\left.\psi_{j l}^{ \pm}\right|_{z=0} ;
\end{gathered}
$$
constitutes the major computational effort in evaluating the second-order exciting forces. In the present calculation, the entire free surface is divided into two regions, an interior, near-field region $S_{f_{1}}$ encompassing the structures and bounded by a fictitious circular boundary $C_{R}$ situated at $r=R$ and an exterior, far-field region $S_{f_{2}}$ extending from $r=R$ to infinity. The partition of the computational domains on the free surface is shown in Fig. 2. In Fig. 2, $C_{\infty}$ is a fictitious boundary whose radius tends to infinity. The integral over the whole free surface is accordingly divided into two parts

$$
\left\{\begin{array}{c}
f_{j l, f, x}^{(2) \pm} \\
f_{j l, f, y}^{(2) \pm}
\end{array}\right\}=\left\{\begin{array}{c}
f_{j l, f_{1}, x}^{(2) \pm} \\
f_{j l, f_{1}, y}^{(2) \pm}
\end{array}\right\}+\left\{\begin{array}{c}
f_{j l, f_{2}, x}^{(2) \pm} \\
f_{j l, f_{2}, y}^{(2) \pm}
\end{array}\right\}
$$

By the integration in the circumferential direction and utilization of the orthogonality of Fourier modes, the free surface integral over the near-field region can be reduced to a series of radial integrals. Then the resulting expression for the integral over the first region can be written as:

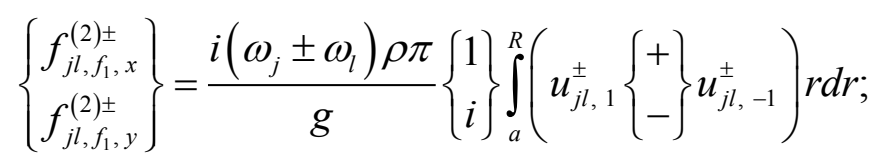

where 


$$
u_{j l, k}^{-}=\sum_{m=-\infty}^{+\infty}\left\{\frac{1}{2} i\left(\omega_{j}-\omega_{l}\right)\left(\frac{\partial \varphi_{j, m}^{(1)}}{\partial r} \frac{\partial \varphi_{l, m-k}^{(1) *}}{\partial r}-\frac{\partial \varphi_{j, I, m}^{(1)}}{\partial r} \frac{\partial \varphi_{l, I, m-k}^{(1) *}}{\partial r}\right)\right.
$$

$$
\left.-\frac{1}{2} i\left[\frac{\omega_{j} \alpha_{l j}-\omega_{l} \alpha_{j l}}{2}-\left(\omega_{j}-\omega_{l}\right) \frac{m(m-k)}{r^{2}}\right]\left(\varphi_{j, m}^{(1)} \varphi_{l, m-k}^{(1) *}-\varphi_{j, I, m}^{(1)} \varphi_{l, I, m-k}^{(1) *}\right)\right\}\left.\psi_{j l}^{ \pm}\right|_{z=0} .
$$

339

340

352 in which

where

For the numerical evaluation of the line integrals in the above equations, Romberg quadrature is used to control the accuracy.

For the calculation in the second region, the contributions from the evanescent modes to the assisting radiation potential are neglected. After applying the integration by parts and using the Bessel differential equation, the integral can be transformed to the form released from derivatives plus some residuals. Then the resulting expression for the integral over the far field can be written as:

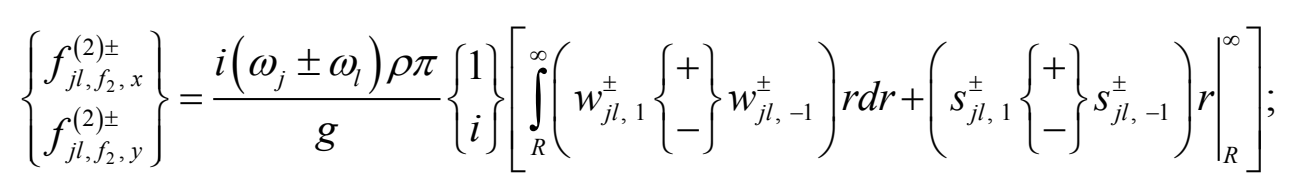

$$
w_{j l, k}^{+}=\left.\frac{i B_{j l, 0}^{+}}{4} \frac{\omega_{j} c_{l j}+\omega_{l} c_{j l}-\left(\kappa_{j l}^{+}\right)^{2}\left(\omega_{j}+\omega_{l}\right)}{\kappa_{j l}^{+} H_{1}^{\prime}\left(\kappa_{j l}^{+} a\right)} \sum_{m=-\infty}^{+\infty}\left(\varphi_{j, m}^{(1)} \varphi_{l,-m+k}^{(1)}-\varphi_{j, I, m}^{(1)} \varphi_{l, I,-m+k}^{(1)}\right) H_{1}\left(\kappa_{j l}^{+} r\right)\right|_{z=0} ;
$$

$$
w_{j l, k}^{-}=\left.\frac{i B_{j l, 0}^{-}}{4} \frac{\omega_{j} c_{l j}-\omega_{l} c_{j l}-\left(\kappa_{j l}^{-}\right)^{2}\left(\omega_{j}-\omega_{l}\right)}{\kappa_{j l}^{-} H_{1}^{\prime}\left(\kappa_{j l}^{-} a\right)} \sum_{m=-\infty}^{+\infty}\left(\varphi_{j, m}^{(1)} \varphi_{l, m-k}^{(1) *}-\varphi_{j, I, m}^{(1)} \varphi_{l, I, m-k}^{(1) *}\right) H_{1}\left(\kappa_{j l}^{-} r\right)\right|_{z=0},
$$

$$
c_{j l}=k_{j}^{2} \tanh ^{2} k_{j} d+2 k_{j} k_{l} \tanh k_{j} d \tanh k_{l} d+k_{l}^{2},
$$

354 and

355

$$
\begin{gathered}
S_{j l, k}^{+}=\frac{i B_{j l, 0}^{+}}{4} \frac{\omega_{j}+\omega_{l}}{\kappa_{j l}^{+} H_{1}^{\prime}\left(\kappa_{j l}^{+} a\right)} \sum_{m=-\infty}^{+\infty}\left[-\kappa_{j l}^{+}\left(\varphi_{j, m}^{(1)} \varphi_{l,-m+k}^{(1)}-\varphi_{j, I, m}^{(1)} \varphi_{l, I,-m+k}^{(1)}\right) H_{1}^{\prime}\left(\kappa_{j l}^{+} r\right)\right. \\
\left.+\left(\frac{\partial \varphi_{j, m}^{(1)}}{\partial r} \varphi_{l,-m+k}^{(1)}+\varphi_{j, m}^{(1)} \frac{\partial \varphi_{l,-m+k}^{(1)}}{\partial r}-\frac{\partial \varphi_{j, I, m}^{(1)}}{\partial r} \varphi_{l, I,-m+k}^{(1)}-\varphi_{j, I, m}^{(1)} \frac{\partial \varphi_{l, I,-m+k}^{(1)}}{\partial r}\right) H_{1}\left(\kappa_{j l}^{+} r\right)\right]\left.\right|_{z=0} ;
\end{gathered}
$$




$$
\begin{gathered}
S_{j l, k}^{-}=\frac{i B_{j l, 0}^{-}}{4} \frac{\omega_{j}-\omega_{l}}{\kappa_{j l}^{-} H_{1}^{\prime}\left(\kappa_{j l}^{-} a\right)} \sum_{m=-\infty}^{+\infty}\left[-\kappa_{j l}^{-}\left(\varphi_{j, m}^{(1)} \varphi_{l, m-k}^{(1) *}-\varphi_{j, I, m}^{(1)} \varphi_{l, I, m-k}^{(1) *}\right) H_{1}^{\prime}\left(\kappa_{j l}^{-} r\right)\right. \\
\left.+\left(\frac{\partial \varphi_{j, m}^{(1)}}{\partial r} \varphi_{l, m-k}^{(1) *}+\varphi_{j, m}^{(1)} \frac{\partial \varphi_{l, m-k}^{(1) *}}{\partial r}-\frac{\partial \varphi_{j, I, m}^{(1)}}{\partial r} \varphi_{l, I, m-k}^{(1) *}-\varphi_{j, I, m}^{(1)} \frac{\partial \varphi_{l, I, m-k}^{(1) *}}{\partial r}\right) H_{1}\left(\kappa_{j l}^{-} r\right)\right]\left.\right|_{z=0} .
\end{gathered}
$$

After substituting the Hankel's asymptotic expansions into the integrand, the line integral in Eq. (46) whose integrand contains three triple products of Hankel functions can be represented by the summations of polynomials of various orders. Eq. (46) can then be explicitly evaluated. In both the sum- and difference-frequency analysis, the region $S_{f_{1}}$ is extended by a minimum radial distance of $a+5 d$. To control the accuracy of the free-surface integral, an adaptive scheme is then adopted in which the extension of the region $S_{f_{1}}$ is progressively increased until the convergence is achieved.

After evaluating the body-surface and free-surface integrals, the total second-order wave force can be determined by the summation of various components:

$$
\left\{\begin{array}{l}
f_{j l, x}^{(2) \pm} \\
f_{j l, y}^{(2) \pm}
\end{array}\right\}=\left\{\begin{array}{l}
f_{j l, q, x}^{(2) \pm} \\
f_{j l, q, y}^{(2) \pm}
\end{array}\right\}+\left\{\begin{array}{c}
f_{j l, b i, x}^{(2) \pm} \\
f_{j l, b i, x}^{(2) \pm}
\end{array}\right\}+\left\{\begin{array}{c}
f_{j l, f, x}^{(2) \pm} \\
f_{j l, f, y}^{(2) \pm}
\end{array}\right\},
$$

in which

$$
\left\{\begin{array}{l}
f_{j l, b i, x}^{(2) \pm} \\
f_{j l, b i, y}^{(2) \pm}
\end{array}\right\}=\left\{\begin{array}{l}
f_{j l, b, x}^{(2) \pm} \\
f_{j l, b, y}^{(2) \pm}
\end{array}\right\}+\left\{\begin{array}{l}
f_{j l, I, x}^{(2) \pm} \\
f_{j l, I, y}^{(2) \pm}
\end{array}\right\} .
$$

\section{Convergence Test and Validation}

The convergence of the present solution depends on both the number of Fourier modes and the number of eigenmodes. In the numerical algorithm, the Fourier series of the first-order potential, Eq. (10), is approximated by $2 M+1$ terms. In addition, the infinite series of the assisting radiation potential, Eq. (31), is approximated by $N+1$ terms. To check the convergence characteristics of the present solution with respect to the number of Fourier modes and the number of eigenmodes, calculations are carried out for a bottom-mounted vertical cylinder of $d / a=4$. Tables 1 and 2 list the dimensionless sum- and difference-frequency surge force for three combinations of incident wave frequencies with $\beta_{j}=\pi / 4$ and $\beta_{l}=0$. The results in Table 1 are listed 
as a function of $N$ with a constant value of $M=15$, while those in Table 2 are corresponding to a variable $M$ and a constant value of $N=100$. In Tables 1 and 2, $v_{j}$ is the deep-water wave number and defined as $\omega_{j}^{2} / g$. Hereinafter, the factors $\rho g a A_{j} A_{l}$ and $\rho g a A_{j} A_{l}^{*}$ are used to nondimensionalize the sum- and differencefrequency forces and the constituent components respectively, and the denotations $\mathbf{f}_{j l}^{ \pm}$, $\mathbf{f}_{j l, q}^{ \pm}, \mathbf{f}_{j l, b i}^{ \pm}$and $\mathbf{f}_{j l, f}^{ \pm}$are used to represent the dimensionless sum- and differencefrequency wave forces and the force components. From Table 1, it can be seen that, for the sum-frequency results, the discrepancies only occur at the fourth decimal place when $N \geq 100$. Moreover, $N=100$ is sufficient for 4 significant decimals of accuracy for the difference-frequency results. From Table 2 it can be seen that, $M=15$ is sufficient for 4 significant decimals of accuracy for both the sum- and differencefrequency results. Hence $M=15$ and $N=100$ are used in all subsequent computations. Above results suggest that the present solution possesses good convergence characteristics.

In order to confirm the validity of the present semi-analytical model, a comparison with the published results is made. The comparison concerns the case that dual waves of different frequencies but the same heading $\left(\beta_{j}=\beta_{l}=0\right)$ act on a bottom-mounted vertical cylinder. Table 3 shows the dimensionless magnitude of the second-order sumand difference-frequency horizontal wave forces for various combinations of wave frequencies. The results of the sum- and difference-frequency wave force are presented in Tables 3(a) and 3(b) respectively. In addition, in Tables 3(a) and 3(b), the upper and lower matrices present the results corresponding to $d / a=1$ and 4 respectively. In Table 3(a), for each wave frequency pair, the results published by Kim and Yue (1990) and Eatock and Huang (1997) are listed in the first and second rows respectively and the present results are listed in the third row. Kim and Yue (1990)'s solution and Eatock and Huang (1997)'s solution are both obtained based on the direct evaluation of the second-order velocity potential. In Table 3(a), for the wave frequency combination of $\left(v_{j} a, v_{l} a\right)=(2.0,2.0)$ with $d / a=4$, the results based on Eatock and Huang (1997)' solution and the present solution are 3.507 and 3.506 respectively, which agree very 
well. While that based on Kim and Yue (1990)'s solution is 3.052. The cause for the substantial discrepancy is not clear. For other wave frequency combinations, the results based on different methods are close to each other. In Table 3(b), for each wave frequency pair, Kim and Yue (1990)'s results are listed in the first row and the present results are listed in the second row. Comparison confirms the good agreement between the present predictions and those based on Kim and Yue (1990)'s solution for all the wave frequency combinations.

To check whether the present solution converges to that of unidirectional waves as the directional spreading decreases, calculation has been performed for the sum- and difference-frequency surge forces on a vertical cylinder of $d / a=4$ for three different wave frequency combinations. The results are presented in Fig. 3 and plotted as a function of $\beta_{j}$, with $\beta_{l}$ fixed at $\beta_{l}=0$. In addition, in Fig. 3, the filled symbols that appear at $\beta_{j}=0$ represent the results based on Kim and Yue (1990)'s solution. From Fig. 3, it can be seen that as $\beta_{j}$ decreases, the present results gradually converge to Kim and Yue (1990)'s results. It suggests that the present solution converges uniformly to that of unidirectional waves as the directional spreading decreases.

To provide a further check on the validity of the present method, another set of comparison concerning the action of dual waves with different frequencies ( $\left.\Delta v a=v_{j} a-v_{l} a=0.2\right)$ and different headings $\left(\beta_{j}=\pi / 4\right.$ and $\left.\beta_{l}=0\right)$ is made. The dimensionless magnitude of the second-order sum- and difference-frequency wave forces corresponding to $d / a=2$ and 4 is illustrated in Figs. 4 and 5. In these figures, the results are plotted as the functions of dimensionless mean wave frequency $v_{m} a=\left(v_{j}+v_{l}\right) a / 2$. The present results together with those based on a higher-order boundary element method (HOBEM) (Teng and Eatock Taylor, 1995) are both shown. For the results from HOBEM, 256 quadratic elements were used in each quadrant (96 elements on the body surface and 160 elements on the water plane area) for $d / a=2$, and 320 quadratic elements were used in each quadrant (160 elements on the body surface and 160 elements on the water plane area) for $d / a=4$. Advantage was taken of the two planes of symmetry. It can be seen that good agreement is obtained between the 
present results and the boundary element results, which further confirms the validity of the present method.

\section{Numerical Results and Discussion}

The frequencies and headings of dual waves can both affect the magnitude of the wave force. In order to illustrate this, calculations are carried out for various combinations of wave frequencies and headings, and the results are presented in this section.

The dimensionless magnitude of the total sum- and difference-frequency surge forces for a vertical cylinder of $d / a=4$ is presented in Table 4 . The results are given for four different combinations of wave heading, in which $\beta_{l}$ is fixed at 0 and $\beta_{j}$ is varied as $\beta_{j}=\pi / 4, \pi / 2,3 \pi / 4$ and $\pi$. The dimensionless frequency range considered is $1 \leq v_{j} a$, $v_{l} a \leq 2$ with an equal spacing of 0.2 . In Table 4 , the results for the sum- and differencefrequency problem are shown in the upper right and lower right triangle matrix respectively. In addition, the sum- and different-frequency results with $\beta_{j}=0$ can be found in the lower left triangle matrix of Table 3(a) and Table 3(b) respectively.

From the results in the upper right triangle matrix in Table 4 and those in the lower left triangle matrix in Table 3(a), the effects of wave directionality on the sumfrequency surge force is clearly observed. Except the case of $\beta_{j}=\pi / 2$, the magnitude of $f_{j l, x}^{+}$increases gradually with increasing the mean wave frequency at a fixed frequency difference. Along the diagonal, the sum-frequency results with $\beta_{j}=\pi$ vanish due to the symmetry of the body. Off the diagonal, the sum-frequency results with $\beta_{j}=\pi$ can be several times as large as those with other wave heading combinations. It indicates that the sum-frequency surge force can be largely amplified when two waves approach the body from opposite directions.

From the results in the lower left triangle matrix in Table 4 and those in the lower left triangle matrix in Table 3(b), obvious difference between the difference-frequency results with different wave heading combinations can be observed. Except the case of $\beta_{j}=\pi / 2$, the magnitude of $f_{j l, x}^{-}$generally increases with increasing the frequency 
difference at a fixed mean wave frequency. Meanwhile, for the wave frequency combination of $\left(v_{j} a, v_{l} a\right)=(1.0,1.0)$, the case of $\beta_{j}=\pi$ gives the largest difference-frequency wave force among the five different wave heading combinations; while for other wave frequency combinations, the case with $\beta_{j}=0$ gives the largest results. For the difference-frequency problem, the largest results are given by the cases of $\beta_{j}=0$ or $\pi$. This can be attributed to the large projected area of wave action when the waves travel along the positive and negative $x$-axis.

The dimensionless magnitude of the sum- and difference-frequency sway forces for a vertical cylinder of $d / a=4$ is presented in Table 5 . As the sway force corresponding to $\beta_{j}=\pi$ vanishes as expected, the results are only presented for $\beta_{j}=\pi / 4, \pi / 2$ and $3 \pi / 4$. From Table 5 , it is found that the general behavior of the sway force is different to the surge force. Both of the sum- and difference-frequency sway forces do not exhibit a clearly discernable trend, and this may be due to the possible phase cancellations among the contributions from their constituent components. Moreover, it is observed that the wave directionality can obviously affect the sway force. For the differencefrequency problem, the $j$ th wave component traveling along the positive $y$-axis $\left(\beta_{j}=\right.$ $\pi / 2$ ) gives the most pronounced results. However, this remark is not valid for the sumfrequency problem. It can be noted that the sum-frequency sway force with $\beta_{j}=3 \pi / 4$ are significantly larger in magnitude than those with other wave heading combinations for each frequency pair.

In Table 6, the dimensionless magnitude of constituent components of the sum- and difference-frequency surge forces is given for a vertical cylinder of $d / a=4$ for $\beta_{j}=$ $\pi / 4$ and $\beta_{l}=0$. The dimensionless frequency range considered is $1 \leq v_{j} a, v_{l} a \leq 2$ with an increment of 0.2 . The corresponding results of the total force have been presented in Table 4. In Table 6, the results corresponding to the sum-frequency problem are shown in the upper right triangle matrix. It can be seen that the force component due to the free-surface integral, $f_{j l, f, x}^{+}$, is dominated for each frequency pair. It is also noted that the contribution from the force component, $f_{j l, b i, x}^{+}$, is almost negligible in the frequency range considered. As $f_{j l, f, x}^{+}$and the force component due to first-order 
interactions, $f_{j l, q, x}^{+}$, are generally out of phase, the magnitude of the total force is smaller than that of $f_{j l, f, x}^{+}$. The lower left triangle matrix in Table 6 contains the results corresponding to the difference-frequency problem. For a fixed mean frequency and increasing frequency difference, the force components $f_{j l, b i, x}^{-}$and $f_{j l, f, x}^{-}$both increase rapidly in magnitude starting from zero on the diagonal; while $f_{j l, q, x}^{-}$ increases with a much milder rate. Near the diagonal, the total force is largely due to the quadratic effects of the first order potentials; while for larger frequency differences, the contribution due to the second-order velocity potential becomes more important.

The dimensionless magnitude of constituent components of the sum- and differencefrequency sway forces is shown in Table 7. The corresponding results for the total force have been shown in Table 5. The force component due to the free-surface integral, $f_{j l, f, y}^{+}$, dominates the total sum-frequency sway force regardless of the wave frequency combination. Meanwhile, the contribution from the force component $f_{j l, b i, y}^{+}$is relatively less important compared to other force components. For the differencefrequency problem, the computationally difficult free-surface integral term, $f_{j l, f, y}^{-}$, is much smaller in magnitude than $f_{j l, q, y}^{-}$and $f_{j l, b i, y}^{-}$. Near the diagonal, the force component due to the first-order interactions, $f_{j l, q, y}^{-}$, dominates the total differencefrequency sway force. As the frequency difference increases, the contribution from $f_{j l, b i, y}^{-}$becomes more pronounced. When the dimensionless frequency difference, $v_{j} a-v_{l} a$, exceeds $0.4, f_{j l, b i, y}^{-}$dominates the total force.

As the main excitations to some important phenomena, such as slow drift and springing, the sum- and difference-frequency wave forces are the critical input to the motion simulations which are important for the design. As illustrated in Section 3, evaluation of these non-linear wave forces requires the complicated formulation and intensive computational effort. The complexity in solving the sum- and differencefrequency wave forces motivates us to make some simplifications on the calculation of those forces. By assuming that the incident waves in the multi-directional seas are narrow-banded in both the frequency and directional spreading, these forces are developed by means of a power expansion with respect to the frequency difference, 
$\Delta \omega=\omega_{j}-\omega_{l}$, and heading difference, $\Delta \beta=\beta_{j}-\beta_{l}$. The following equations can then be obtained

$$
\begin{gathered}
\mathbf{f}_{j l}^{ \pm}\left(\omega_{j}, \omega_{l} ; \beta_{j}, \beta_{l}\right)=\mathbf{f}_{j l}^{ \pm}\left(\omega_{j}, \omega_{l} ; \hat{\beta}, \hat{\beta}\right)+O(\Delta \beta) ; \\
\mathbf{f}_{j l}^{ \pm}\left(\omega_{j}, \omega_{l} ; \beta_{j}, \beta_{l}\right)=\mathbf{f}_{j l}^{ \pm}\left(\hat{\omega}, \hat{\omega} ; \beta_{j}, \beta_{l}\right)+O(\Delta \omega) ; \\
\mathbf{f}_{j l}^{ \pm}\left(\omega_{j}, \omega_{l} ; \beta_{j}, \beta_{l}\right)=\mathbf{f}_{j l}^{ \pm}(\hat{\omega}, \hat{\omega} ; \hat{\beta}, \hat{\beta})+O(\Delta \omega)+O(\Delta \beta),
\end{gathered}
$$

in which $\hat{\omega}=\left(\omega_{j}+\omega_{l}\right) / 2$ and $\hat{\beta}=\left(\beta_{j}+\beta_{l}\right) / 2$. According to Eq. (49), three approximations on the calculation of the sum- and difference-frequency wave loads can be obtained. In the first approximation, the wave frequency is treated exactly, while approximation is made on the wave heading and only the zeroth-order terms with respect to $\Delta \beta$ are retained. Then the bi-chromatic bi-directional problem is approximated by the bi-chromatic but unidirectional one with $\beta_{j}=\beta_{l}=\hat{\beta}$. In the second approximation, the wave heading is treated exactly, while the higher order terms with respect to $\Delta \omega$ are neglected. Then the bi-chromatic bi-directional problem is transferred to the bi-directional but monochromatic one with $\omega_{j}=\omega_{l}=\hat{\omega}$. In the third approximation, only the zeroth-order terms with respect to $\Delta \omega$ and $\Delta \beta$ are retained. Then the bi-chromatic bi-directional problem is replaced by the monochromatic unidirectional one with $\omega_{j}=\omega_{l}=\hat{\omega}$ and $\beta_{j}=\beta_{l}=\hat{\beta}$.

To assess the relative superiority of one approximation over the others, calculation is carried out based on the different approximations and then the results are compared with those based on the complete solution, in which the frequencies and headings of dual waves are treated exactly. Again, a bottom-mounted cylinder of $d / a=4$ is considered as an example. During the calculation, the wave heading of one wave is fixed at $\beta_{l}=0$, and that of the other one varies between $\beta_{j}=\pi / 18$ and $\pi / 9$. As both the waves travel along the direction close to the positive $x$-axis, only the results for the total surge force and its constituent components are shown here. Figs. 6-9 plot the dimensionless magnitude of the sum- and difference-frequency surge forces and the constituent components as the functions of dimensionless mean frequency $v_{m} a=\left(v_{j} a+v_{l} a\right) / 2$. In these figures, the results referred as 'complete' are obtained 
based on the complete solution, while those referred as 'A1', 'A2' and 'A3' are obtained based on the three approximations respectively.

Fig. 6 shows the results for the sum-frequency problem with the dimensionless frequency difference, $\Delta v a$, fixed at 0.1 and the wave heading difference, $\Delta \beta$, equal to $\pi / 18$. In Fig. 6(a), the results based on the complete solution agree well with those based on the approximation which exactly treats the wave heading. In the low frequency region, these results decay quickly as $v_{m} a$ increases until vanish. After that, they first increase gradually as $v_{m} a$ increases until reach the peak value and then decrease gradually as $v_{m} a$ increases further. Other approximate results in Fig. 6(a) agree well with each other; however, they follow an obviously different trend with $v_{m} a$ and continue to decrease as $v_{m} a$ increases. In Figs. 6(b) and 6(c) different results agree well with each other, and in this case, $\Delta v a$ and $\Delta \beta$ almost have no effects on the force components $f_{j l, f, x}^{+}$and $f_{j l, q, x}^{+}$. As the contribution from $f_{j l, b i, x}^{+}$to the total force is insignificant, different approximations perform well in predicting the total force in this case and good agreement between different results can be observed from Fig. $6(d)$

Fig. 7 describes the dimensionless magnitude of the sum-frequency surge force and its constituent components with $\Delta v a=0.2$ and $\Delta \beta=\pi / 9$. In Fig. 7(a), the wave heading difference still obviously affects the force component $f_{j l, b i, x}^{+}$. In Fig. 7(b), the approximation which exactly treats the wave frequency in general gives satisfactory predictions. Other approximations tend to overestimate the magnitude of $f_{j l, f, x}^{+}$. In Fig. 7(c), the results based on the approximation that exactly treats the wave heading in general agree with the results based on the complete solution. Other approximations give underestimated predictions when $k a<1.1$, while provide overestimated predictions as $k a$ exceeds 1.1. In Fig. 7(d), the approximation that exactly treats the wave frequency satisfactorily predicts the total force. Meanwhile, other approximations tend to give conservative predictions.

Fig. 8 shows the results for the difference-frequency problem corresponding to $\Delta v a$ $=0.1$ and $\Delta \beta=\pi / 18$. From Figs. $8(\mathrm{a})$ and $8(\mathrm{~b})$, we find that the approximation that 
582

583

584

585

586

587

588

589

590

591

592

593

594

595

596

597

598

599

600

601

602

603

604

605

606

607

608

609

610

exactly treats the wave frequency largely overestimates the magnitude of $f_{j l, b i, x}^{-}$ while slightly underestimates the magnitude of $f_{j l, f, x}^{-}$. Meanwhile, other approximations give zero value results to $f_{j l, b i, x}^{-}$and $f_{j l, f, x}^{-}$, which is due to the fact that in the presence of monochromatic incident waves the maximum contribution of the steady second-order potential to the wave force is of the third order with respect to the wave steepness $\varepsilon$. In Fig. 8(c), different approximations give satisfactory predictions of the magnitude of $f_{j l, q, x}^{-}$. In Fig. 8(d), the results based on the approximation exactly treats the wave headings agree well with those based the complete solution. Meanwhile, if high accuracy is not required, other approximations can give acceptable predictions for the total force.

Fig. 9 describes the dimensionless magnitude of the difference-frequency surge force and its constituent components with $\Delta v a=0.2$ and $\Delta \beta=\pi / 9$. As shown in Figs. 9(a) and 9(b), both the wave frequency difference and wave heading difference obviously affect the force components $f_{j l, b i, x}^{-}$and $f_{j l, f, x}^{-}$. In Fig. 9(c), the approximation that exactly treat the wave heading well predicts the magnitude of $f_{j l, q, x}^{-}$; however, other approximations give overestimated predictions. In Fig. 9(d), different approximate results exhibit the similar trend with $v_{m} a$, and those based on the approximation that exactly treats the wave heading are closer to the results based on the complete solution when compared with other approximations.

\section{Conclusions}

A complete solution is presented for the second-order hydrodynamic forces due to the action of bi-chromatic bi-directional waves on a bottom-mounted, surface-piercing cylinder in the water of uniform finite depth. Semi-analytical formulation for the sumand difference-frequency wave loads is provided. The present solution is validated through a comparison with the results based on other methods. In addition, results are presented for various combinations of wave frequencies and headings. Contributions of its constituent components to the total second-order wave force are discussed. The influence of frequencies and headings of dual waves on the second-order wave force is 
investigated. Furthermore, efforts are devoted to make some simplifications on the calculation of these nonlinear forces, for the cases of small wave frequency difference and wave heading difference. The main conclusions of this study can be summarized as:

1) The validity of the present solution is examined by comparing with the results based on other methods. The comparison shows a favorable agreement between the predictions by different methods.

2) Under the action of dual waves with different frequencies and headings, the freesurface integral term is the dominated contribution to the total sum-frequency wave force among the constituent components. For the difference-frequency problem, the total difference-frequency wave force is largely due to the quadratic effects from the first-order potentials when the frequency difference is small, and the contribution due to the second-order velocity potential gets more pronounced as the frequency difference grows larger.

3) The present results illustrate that the wave directionality can obviously affect the second-order hydrodynamic forces. Large amplification or reduction in magnitude of the second-order wave loads can be induced by including the effects of wave directionality. With the non-zero frequency differences in this study, the sum-frequency surge force can be several times larger when two waves approach the body from the opposite headings than other combinations of wave headings. The assumption of unidirectional waves does not always lead to a safe estimation of the second-order wave loads.

4) The sum- and difference-frequency wave loads can be expressed in a power expansion with respect to the wave frequency difference and wave heading difference both of which are assumed to be small. Then different approximate ways to calculate these forces are obtained. In the sum-frequency problem, the wave length can be much smaller when compared with the characteristic size of the cylinder. It suggests that the wave diffraction effects can be significant in the sum-frequency problem and a small change in the wave number may lead to a considerable disturb on the wave field in the 
vicinity of the structure. Through comparing the approximate results with those based the complete solution, it is found that approximating the bi-chromatic bi-directional problem by the bi-chromatic but unidirectional problem can be an effective simplification in predicting the sum-frequency wave loads on a bottom-mounted cylinder when the wave heading difference is not large. Meanwhile, for the differencefrequency problem, the wave length can be obviously larger than the characteristic size of the cylinder especially when the frequency-difference is small. It suggests that a small change in the wave number may not make considerable effects on the wave field around the structure. Moreover, the comparison reveals that neglecting the effects of wave heading difference tend to overestimate the results of the difference-frequency wave force, while more acceptable results can be obtained through approximating the bi-chromatic bi-directional problem by the bi-directional but monochromatic problem.

\section{Acknowledgment}

The work is financially supported by the National Natural Science Foundation of China (Grant Nos. 51379032, 51479026 and 51679036).

\section{Reference}

[1] Abul-Azm A G, Williams A N. Second-order diffraction loads on arrays of semi-immersed circular cylinders. Journal of Fluids and Structures, 1989, 3(4): 365-387.

[2] Abul-Azm A G, Williams A N. Second-order diffraction loads on truncated cylinders. Journal of Waterway, Port, Coastal, and Ocean Engineering, 1988, 114(4): 436-454.

[3] Chau F P. The second order velocity potential for diffraction of waves by fixed offshore structures. University College London, 1989.

[4] Eatock Taylor R, Hung S M. Second order diffraction forces on a vertical cylinder in regular waves. Applied Ocean Research, 1987, 9(1): 19-30.

[5] Eatock Taylor R, Hung S, Mitchell K. Advances in the prediction of low frequency drift behavior. Proceedings of the 5th International Conference on Behavior of Offshore Structures, Trondheim, Norway, 1988: 651-666.

[6] Eatock Taylor R, Kernot M P. On second order wave loading and response in irregular seas. In Advances in Coastal and Ocean Engineering, Vol. 5, Ed. P. L.-F. Liu, World Scientific $1999,155-212$. 
[7] Ghalayini S A, Williams A N. Nonlinear wave forces on vertical cylinder arrays. Journal of Fluids and Structures, 1991, 5(1): 1-32.

[8] Garrett C J R. Wave forces on a circular dock. Journal of Fluid Mechanics, 1971, 46(1): 129139.

[9] Göteman M. Wave energy parks with point-absorbers of different dimensions. Journal of Fluids \& Structures, 2017, 74: 142-157.

[10] Huang J B, Eatock Taylor R. Semi-analytical solution for second-order wave diffraction by a truncated circular cylinder in monochromatic waves. Journal of Fluid Mechanics, 1996, 319: 171-196.

[11] Kagemoto H, Yue D K P. Interactions among multiple three-dimensional bodies in water waves: an exact algebraic method. Journal of Fluid Mechanics, 1986, 166(166): 189-209.

[12] Kim M H. Difference-frequency wave loads on a large body in multi-directional waves. Applied Ocean Research, 1992, 14(6): 353-370.

[13] Kim M H, Chen W. Slender-body approximation for slowly-varying wave loads in multidirectional waves. Applied Ocean Research, 1994, 16(3): 141-163.

[14] Kim M H. Second-harmonic vertical wave loads on arrays of deep-draft circular cylinders in monochromatic uni- and multi-directional waves. Applied Ocean Research, 1993, 15(5): 245-262.

[15] Kim M H, Yue D K P. The complete second-order diffraction solution for an axisymmetric body, Part 2, Bichromatic incident waves and body motions. Journal of Fluid Mechanics, 1990, 211: 557-593

[16] Lighthill J. Waves and hydrodynamic loading. Proceedings of the 2nd International Conference on the Behaviour of Offshore Structures, London, UK, 1979: 363-370.

[17] Linton C M, Evans D V, et al. The interaction of waves with arrays of vertical circular cylinders. Journal of Fluid Mechanics, 1990, 215(-1): 549-569.

[18] Liu J, Guo A, Li H. Analytical solution for the linear wave diffraction by a uniform vertical cylinder with an arbitrary smooth cross-section. Ocean Engineering, 2016, 126: 163-175.

[19] Malenica Š, Eatock Taylor R, Huang J B. Second-order water wave diffraction by an array of vertical cylinders. Journal of Fluid Mechanics, 1999, 390: 349-373.

[20] Molin B. Second-order diffraction loads upon three-dimensional bodies. Applied Ocean Research, 1979, 1(4): 197-202.

[21] Moubayed W I, Williams A N. Second-order hydrodynamic interactions in an array of vertical cylinders in bichromatic waves. Journal of Fluids and Structures, 1995, 9(1): 61-98.

[22] Newman, J. N. Second-harmonic wave diffraction at large depths. Journal of Fluid Mechanics, 1990, 213(-1): 59-70.

[23] Ogilvie T F. Second Order Hydrodynamic Effects on Ocean Platforms. Proceedings of International Workshop on Ship and Platform Motions. California, USA, 1983. 
[24] Petrauskas C, Liu S V. Springing Force Response of a Tension Leg Platform. Proceedings of 19th Offshore Technology Conference, Houston, USA, 1987: 333-341.

[25] Rahman M, Bhatta D D. Evaluation of added mass and damping coefficient of an oscillating circular cylinder. Applied Mathematical Modelling, 1993, 17(2):70-79.

[26] Rainey R C T. A new equation for calculating wave loads on offshore structures. Journal of Fluid Mechanics, 1989, 204: 295-324.

[27] Renaud M, Rezende F, Waals O, et al. Second-order wave loads on a LNG carrier in multidirectional waves, Proceedings of International Conference on Offshore Mechanics and Arctic Engineering, Estoril, Portugal, 2008, 363-370.

[28] Siddorn P, Taylor R E. Diffraction and independent radiation by an array of floating cylinders. Ocean Engineering, 2008, 35(13): 1289-1303.

[29] Teng B, Eatock Taylor R. New higher-order boundary element methods for wave diffraction/radiation. Applied Ocean Research, 1995, 17(2): 71-77.

[30] Teng B, Kato S. A method for second-order diffraction potential from an axisymmetric body. Ocean Engineering, 1999, 26(12): 1359-1387.

[31] Vazquez J H. Hydrodynamics loads on offshore structures in bichromatic bidirectional seas. University of Houston, 1995.

[32] Wu B J, Zheng Y H, You Y G, et al. On diffraction and radiation problem for two cylinders in water of finite depth. Ocean Engineering, 2006, 33(5): 679-704.

[33] Yeung R W. Added mass and damping of a vertical cylinder in finite-depth waters. Applied Ocean Research, 1981, 3(3): 119-133.

[34] Y1lmaz O, Incecik A. Analytical solutions of the diffraction problem of a group of truncated vertical cylinders. Ocean Engineering, 1998, 25(6): 385-394.

[35] Zheng S M and Zhang Y L. Wave diffraction from a truncated cylinder in front of a vertical wall. Ocean Engineering, 2015, 104: 329-343. 


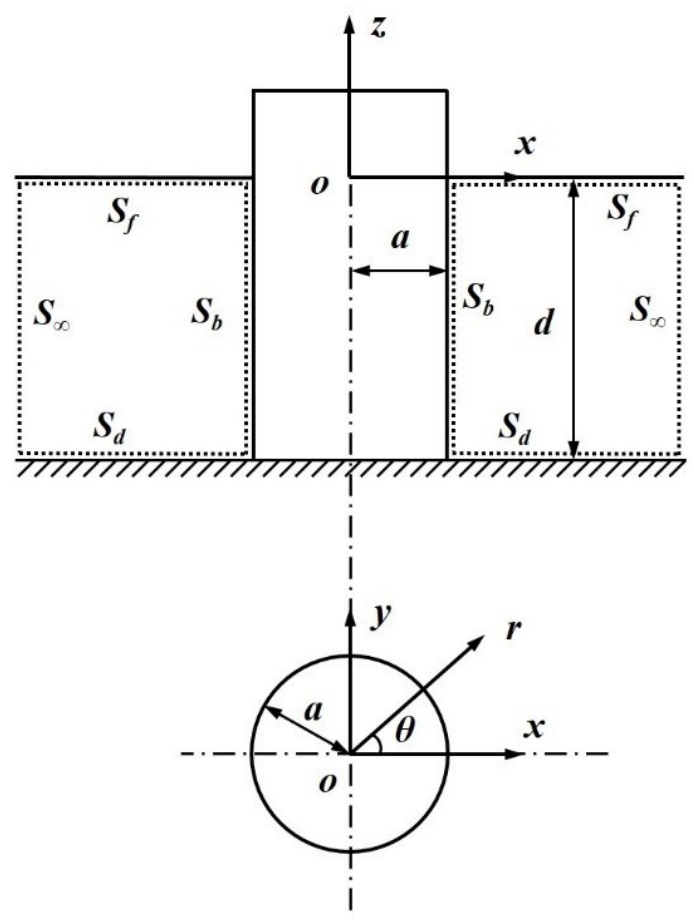

Fig. 1 Definition of the coordinate system

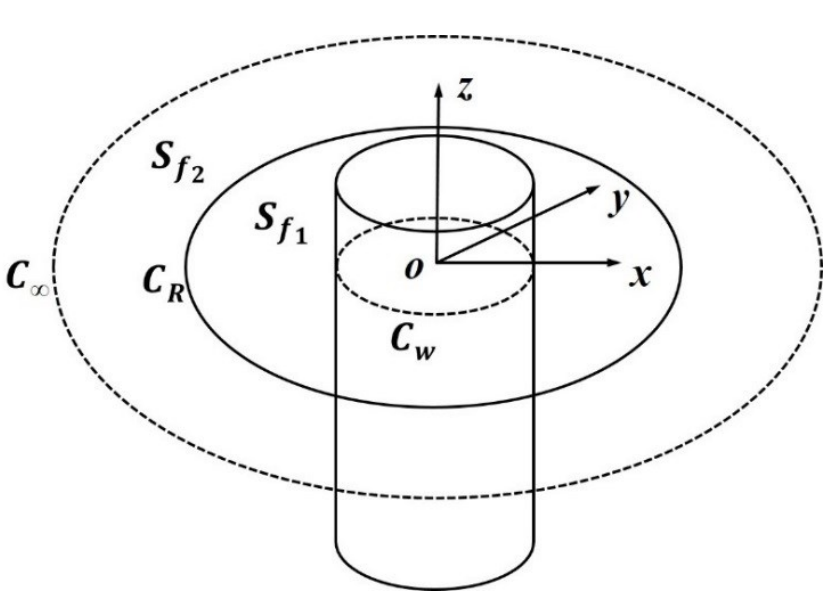

Fig. 2. Partition of the computational domains on the free surface 

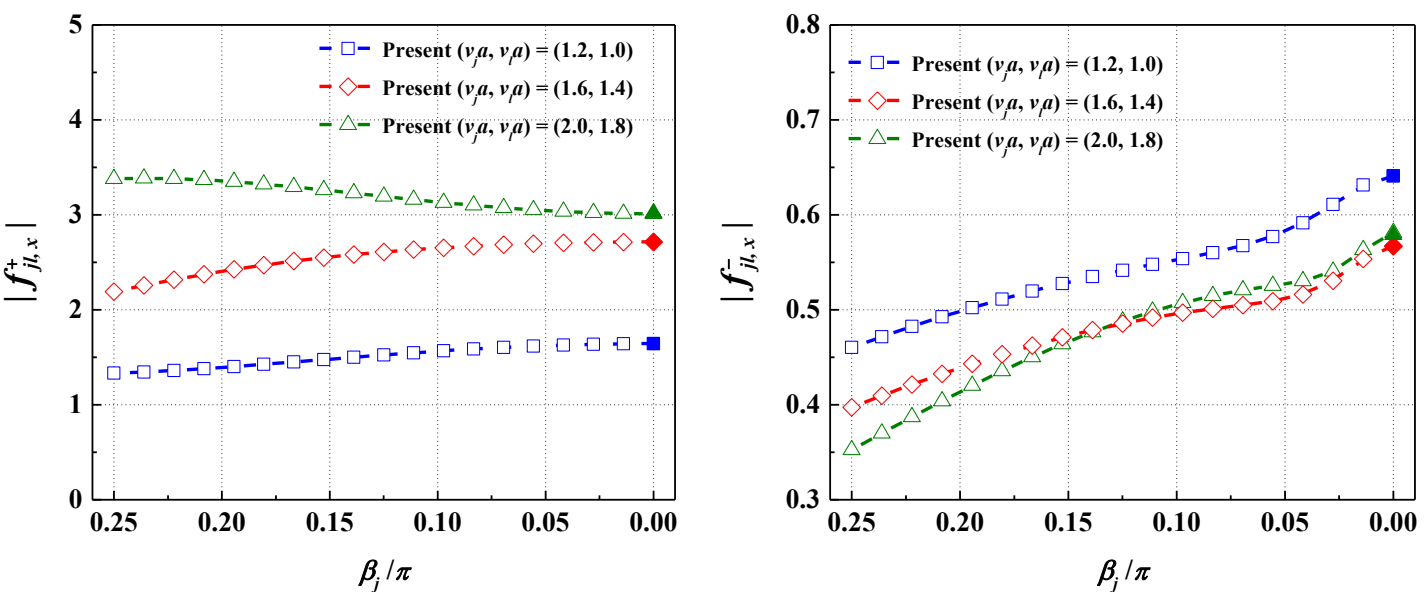

743

744

745

746

747

748

749

750

751

752

753

754

Fig. 3 Dimensionless magnitude of the sum- and difference-frequency surge forces on a vertical cylinder of $d / a=4$ for three different wave frequency combination with $\beta_{l}$ $=0$

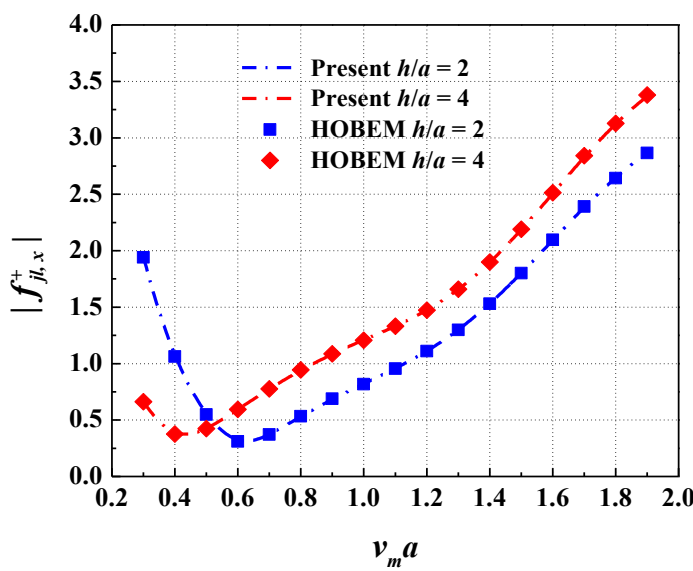

(a) Sum frequency

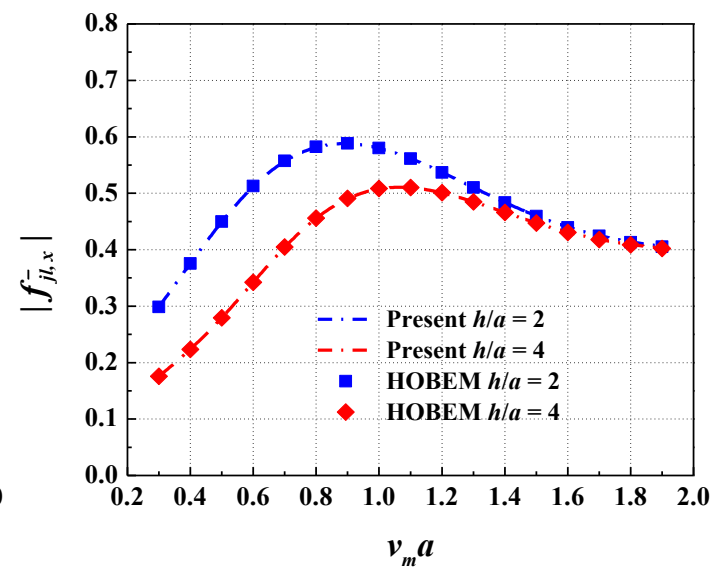

(b) Difference frequency

Fig. 4 Comparison of the dimensionless magnitude of the sum- and differencefrequency surge forces, $f_{j l, x}^{ \pm}$, on a vertical cylinder of $d / a=2$ and 4 with $\Delta v a=0.2$, $\beta_{j}=\pi / 4$ and $\beta_{l}=0$ 


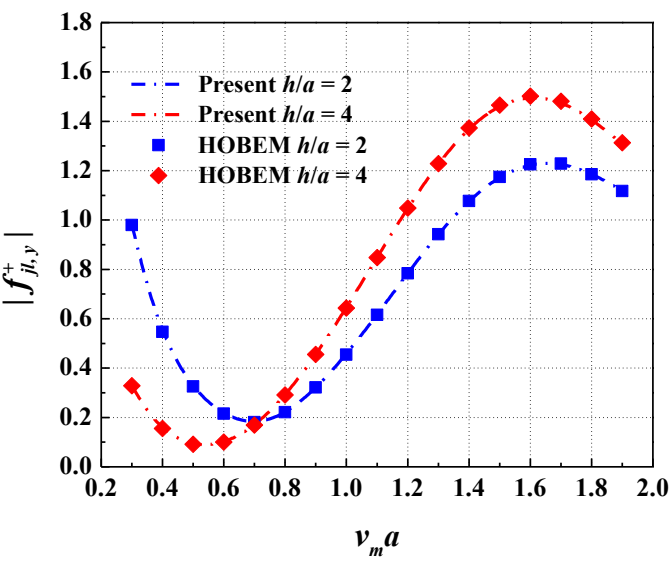

755

756

757

758

759

760 $\beta_{j}=\pi / 4$ and $\beta_{l}=0$

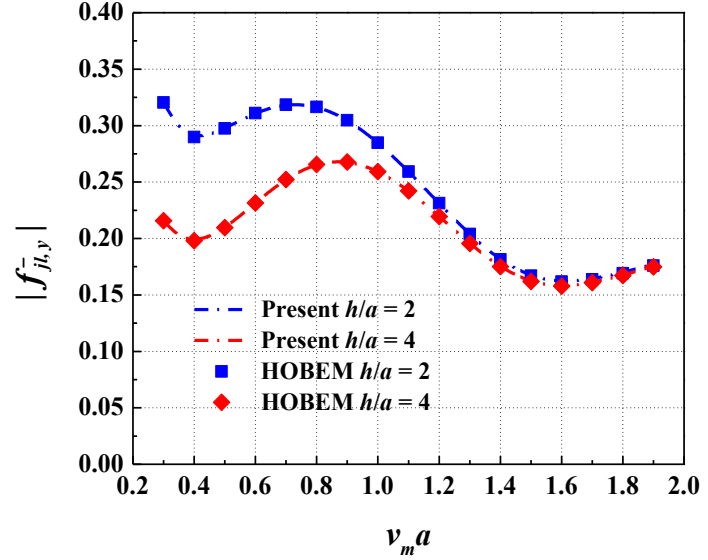

(b) Difference frequency

Fig. 5 Comparison of the dimensionless magnitude of the sum- and differencefrequency sway forces, $f_{j l, y}^{ \pm}$, on a vertical cylinder of $d / a=2$ and 4 with $\Delta v a=0.2$, 


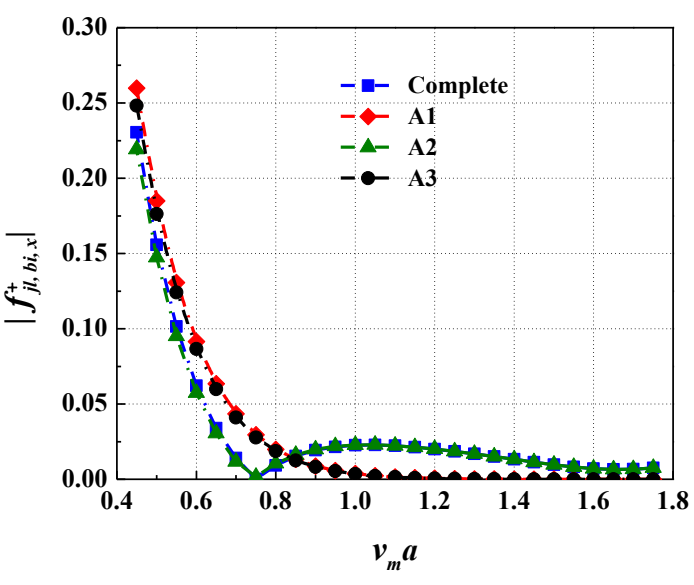

(a) $f_{j l, b i, x}^{+}$

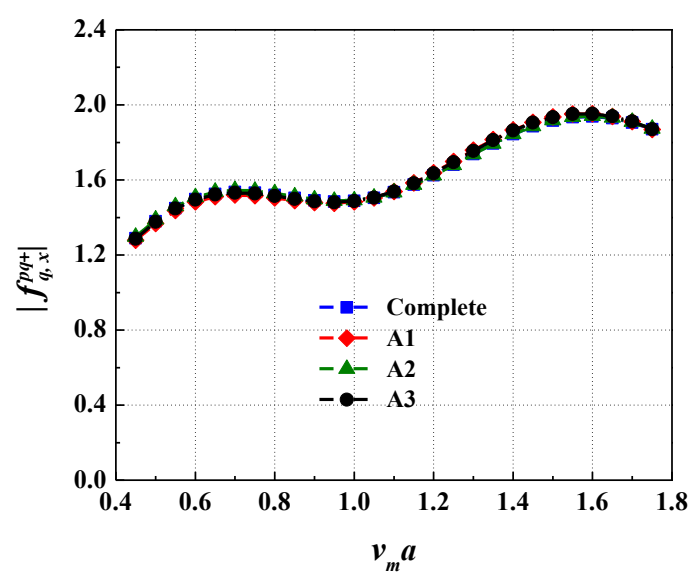

763

764

765

766 767

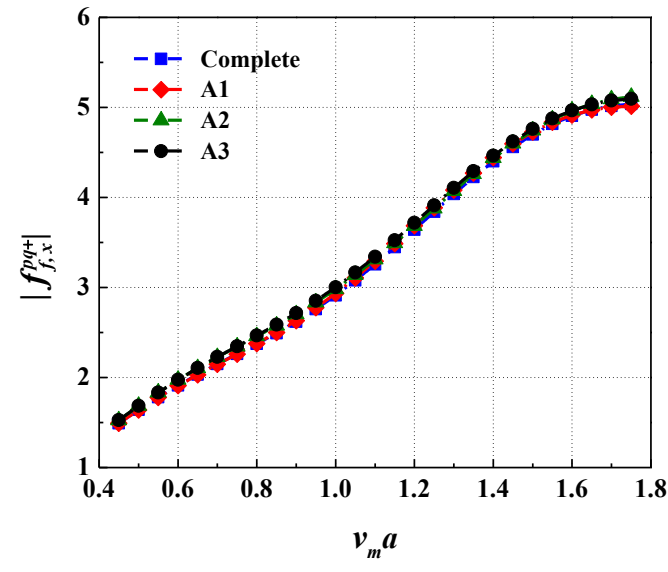

(b) $f_{j l, f, x}^{+}$

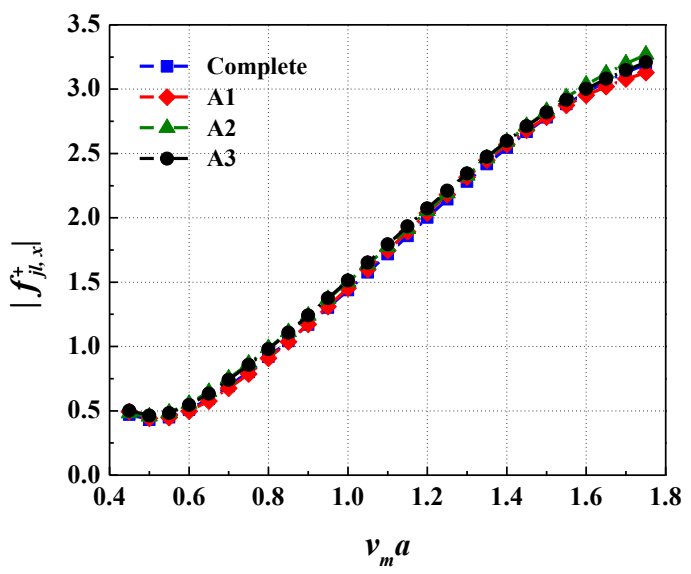

(d) $f_{j l, x}^{+}$

Fig. 6 Dimensionless magnitude of the sum-frequency surge force and its constituent components on a vertical cylinder of $d / a=4$ with $\Delta v a=0.1, \beta_{j}=\pi / 18$ and $\beta_{l}=0$. 


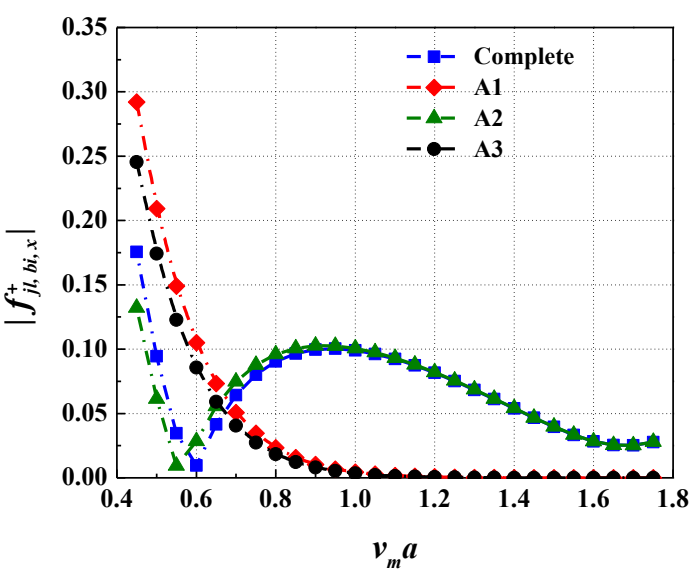

(a) $f_{j l, b i, x}^{+}$

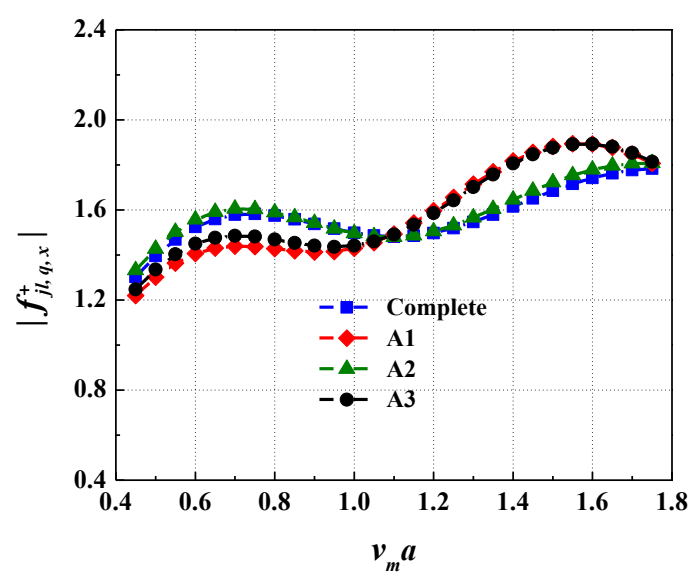

(c) $f_{j l, q, x}^{+}$

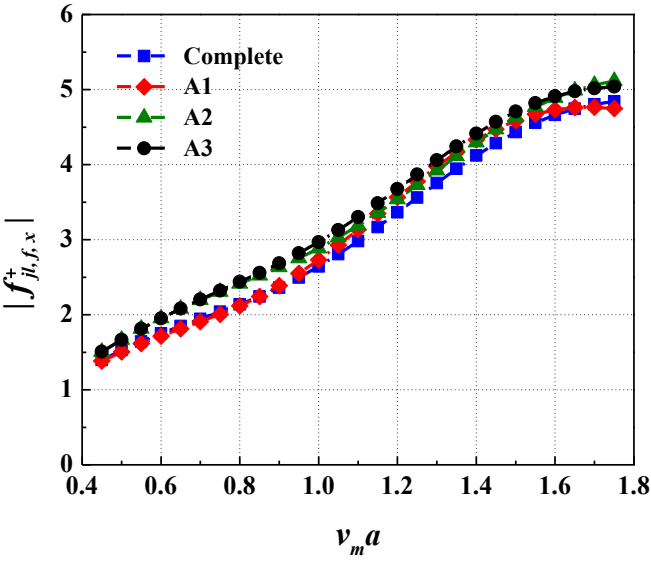

(b) $f_{j l, f, x}^{+}$

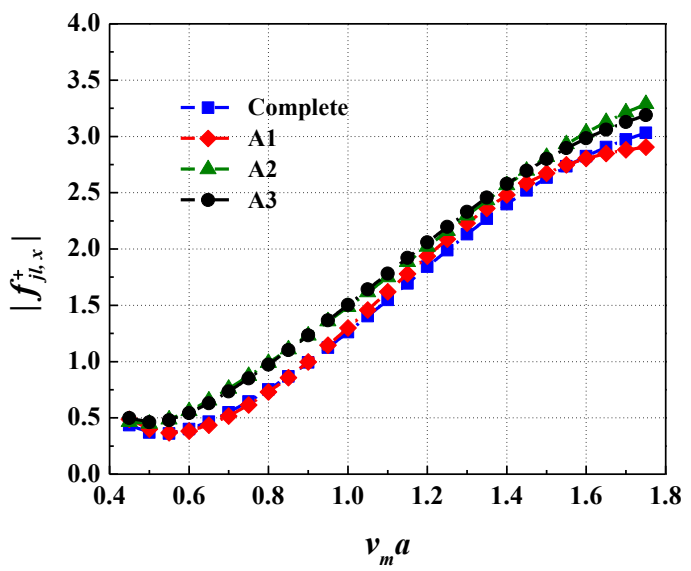

(d) $f_{j l, x}^{+}$

772 Fig. 7 Dimensionless magnitude of the sum-frequency surge force and its constituent 773 components on a vertical cylinder of $d / a=4$ with $\Delta v a=0.2, \beta_{j}=\pi / 9$ and $\beta_{l}=0$. 


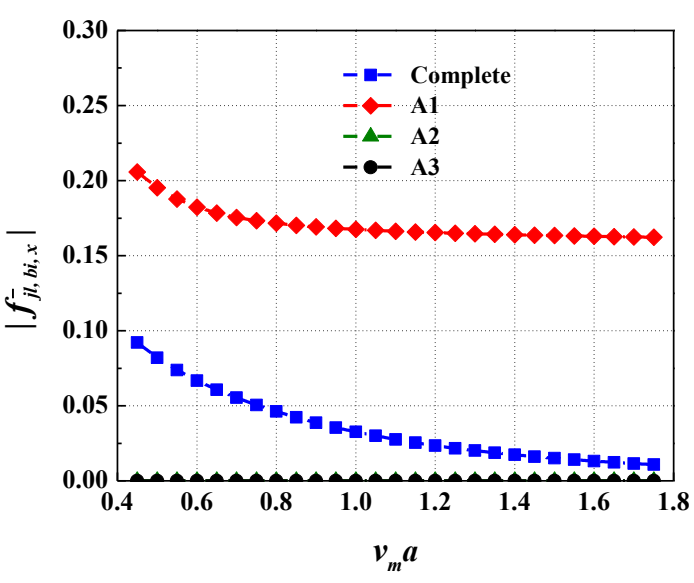

(a) $f_{j l, b i, x}^{-}$

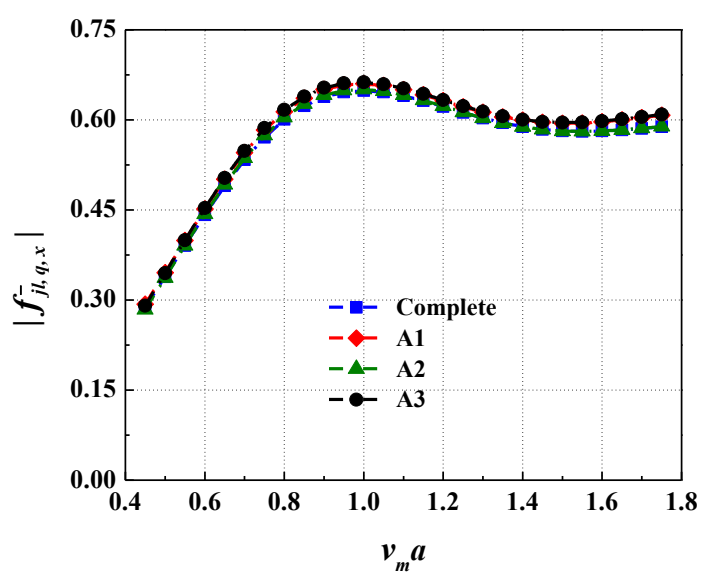

(c) $f_{j l, q, x}^{-}$

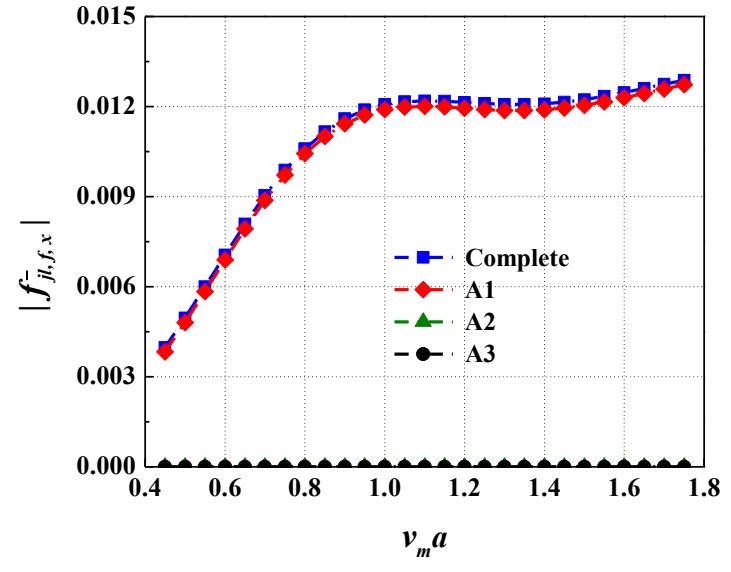

(b) $f_{j l, f, x}^{-}$

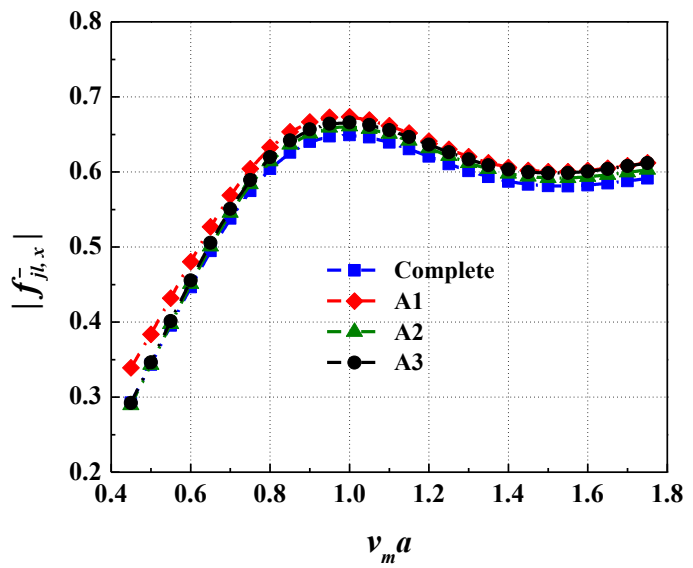

(d) $f_{j l, x}^{-}$

779 Fig. 8 Dimensionless magnitude of the difference-frequency surge force and its 780 constituent components on a vertical cylinder of $d / a=4$ with $\Delta v a=0.1, \quad \beta_{j}=\pi / 18$ and $781 \quad \beta_{l}=0$. 


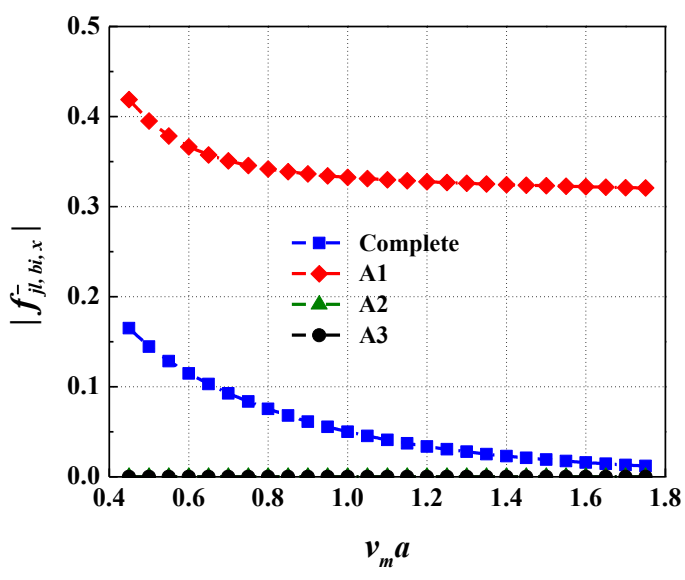

(a) $f_{j l, b i, x}^{-}$

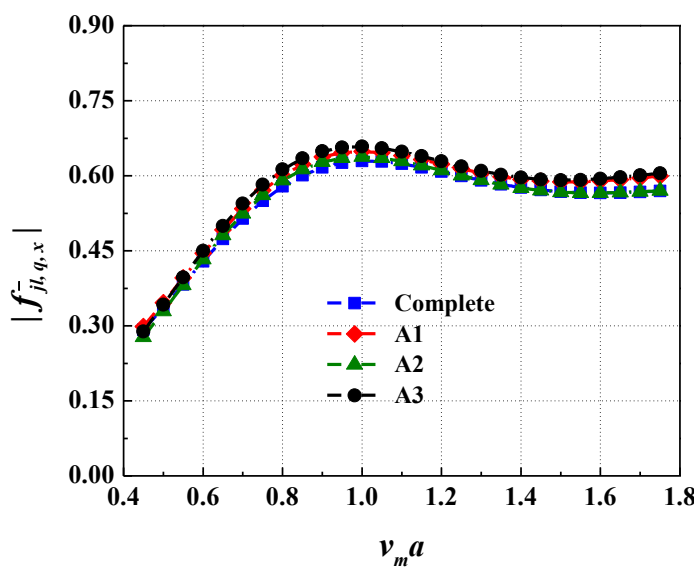

(c) $f_{j l, q, x}^{-}$

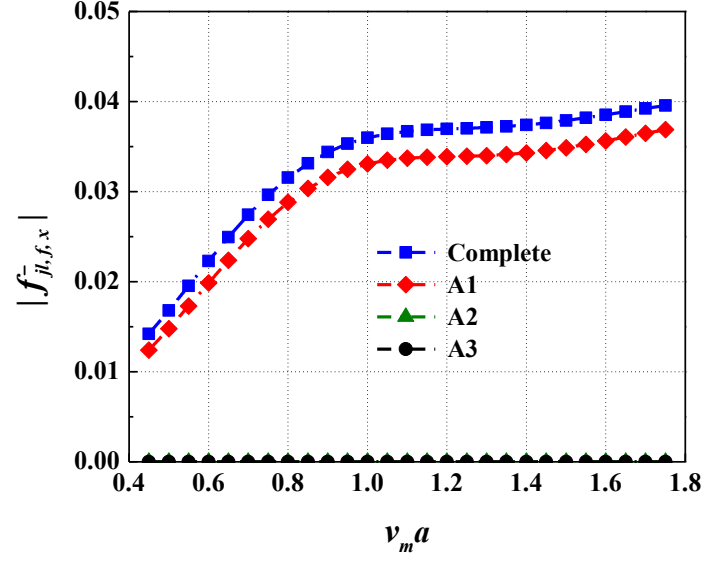

(b) $f_{j l, f, x}^{-}$

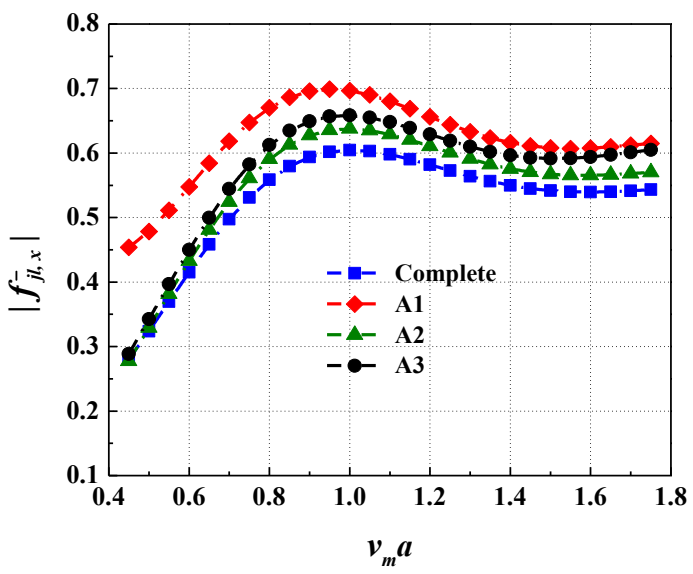

(d) $f_{j l, x}^{-}$

Fig. 9 Dimensionless magnitude of the difference-frequency surge force and its constituent components on a vertical cylinder of $d / a=4$ with $\Delta v a=0.2, \quad \beta_{j}=\pi / 9$ and $\beta_{l}=0$.

790 
791 Table 1 Convergence test on the dimensionless sum- and difference-frequency surge 792 forces, $f_{j l, x}^{ \pm}$, on a vertical cylinder with varying $N\left(M=15, d / a=4, \beta_{j}=\pi / 4\right.$ and $793 \quad \beta_{l}=0$ )

794

(a) Sum-frequency

\begin{tabular}{cccc}
\hline$\left(v_{j} a, v_{l} a\right)$ & $(1.2,1.0)$ & $(1.4,1.0)$ & $(1.6,1.0)$ \\
\hline$N=20$ & $(0.7355,1.1099)$ & $(0.7392,0.7642)$ & $(0.7488,0.4227)$ \\
$N=50$ & $(0.7366,1.1107)$ & $(0.7407,0.7650)$ & $(0.7509,0.4233)$ \\
$N=100$ & $(0.7367,1.1108)$ & $(0.7408,0.7651)$ & $(0.7510,0.4233)$ \\
$N=150$ & $(0.7367,1.1108)$ & $(0.7408,0.7651)$ & $(0.7510,0.4234)$ \\
\hline
\end{tabular}

795

(b) Difference-frequency

\begin{tabular}{cccc}
\hline$\left(v_{j} a, v_{l} a\right)$ & $(1.2,1.0)$ & $(1.4,1.0)$ & $(1.6,1.0)$ \\
\hline$N=20$ & $(0.4930,-0.1316)$ & $(0.4413,-0.2567)$ & $(0.3984,-0.3820)$ \\
$N=50$ & $(0.4930,-0.1316)$ & $(0.4413,-0.2567)$ & $(0.3984,-0.3820)$ \\
$N=100$ & $(0.4930,-0.1316)$ & $(0.4413,-0.2567)$ & $(0.3984,-0.3820)$ \\
$N=150$ & $(0.4930,-0.1316)$ & $(0.4413,-0.2567)$ & $(0.3984,-0.3820)$ \\
\hline
\end{tabular}

796 
797 Table 2 Convergence test on the dimensionless sum- and difference-frequency surge 798 forces, $f_{j l, x}^{ \pm}$, on a vertical cylinder with varying $M\left(N=100, d / a=4, \beta_{j}=\pi / 4\right.$ and $\left.799 \quad \beta_{l}=0\right)$

800

(a) Sum-frequency

\begin{tabular}{cccc}
\hline$\left(v_{j} a, v_{l} a\right)$ & $(1.2,1.0)$ & $(1.4,1.0)$ & $(1.6,1.0)$ \\
\hline$M=3$ & $(0.7383,1.1105)$ & $(0.7421,0.7635)$ & $(0.7517,0.4200)$ \\
$M=10$ & $(0.7367,1.1108)$ & $(0.7408,0.7651)$ & $(0.7510,0.4233)$ \\
$M=15$ & $(0.7367,1.1108)$ & $(0.7408,0.7651)$ & $(0.7510,0.4233)$ \\
$M=20$ & $(0.7367,1.1108)$ & $(0.7408,0.7651)$ & $(0.7510,0.4233)$ \\
\hline
\end{tabular}

801

(b) Difference-frequency

\begin{tabular}{cccc}
\hline$\left.\left(v_{j} a, v_{l} a\right)\right)$ & $(1.2,1.0)$ & $(1.4,1.0)$ & $(1.6,1.0)$ \\
\hline$M=3$ & $(0.4932,-0.1303)$ & $(0.4415,-0.2539)$ & $(0.3985,-0.3769)$ \\
$M=10$ & $(0.4930,-0.1316)$ & $(0.4413,-0.2567)$ & $(0.3984,-0.3820)$ \\
$M=15$ & $(0.4930,-0.1316)$ & $(0.4413,-0.2567)$ & $(0.3984,-0.3820)$ \\
$M=20$ & $(0.4930,-0.1316)$ & $(0.4413,-0.2567)$ & $(0.3984,-0.3820)$ \\
\hline
\end{tabular}

802 
803 Table 3 Comparison of the dimensionless magnitude of the sum- and difference804 frequency horizontal wave forces, $\left|f_{j l, x}^{ \pm}\right|$, on a vertical cylinder for $\beta_{j}=\beta_{l}=0$. The 805 upper right and lower left triangle matrices present results correspond to $d / a=1$ and 4 806 respectively.

807

(a) Sum-frequency

\begin{tabular}{|c|c|c|c|c|c|c|c|}
\hline & & 1.0 & 1.2 & 1.4 & 1.6 & 1.8 & 2.0 \\
\hline \multirow{3}{*}{1.0} & 1.518 & 0.939 & 0.782 & 0.778 & 0.850 & 0.903 & 0.886 \\
\hline & 1.526 & 0.946 & 0.775 & 0.769 & 0.886 & 0.909 & 0.855 \\
\hline & 1.527 & 0.945 & 0.787 & 0.781 & 0.850 & 0.898 & 0.874 \\
\hline \multirow{3}{*}{1.2} & 1.641 & 2.084 & 0.752 & 0.847 & 0.959 & 1.013 & 0.973 \\
\hline & 1.667 & 2.029 & 0.757 & 0.829 & 0.941 & 1.031 & 0.955 \\
\hline & 1.644 & 2.091 & 0.755 & 0.848 & 0.958 & 1.007 & 0.959 \\
\hline \multirow{3}{*}{1.4} & 1.748 & 2.262 & 2.612 & 0.971 & 1.074 & 1.105 & 1.037 \\
\hline & 1.749 & 2.298 & 2.621 & 0.972 & 1.045 & 1.115 & 1.062 \\
\hline & 1.755 & 2.264 & 2.620 & 0.971 & 1.072 & 1.099 & 1.021 \\
\hline \multirow{3}{*}{1.6} & 1.853 & 2.302 & 2.714 & 3.021 & 1.160 & 1.184 & 1.114 \\
\hline & 1.883 & 2.336 & 2.795 & 3.030 & 1.157 & 1.167 & 1.135 \\
\hline & 1.859 & 2.309 & 2.715 & 3.029 & 1.157 & 1.176 & 1.105 \\
\hline \multirow{3}{*}{1.8} & 1.809 & 2.182 & 2.505 & 2.935 & 3.277 & 1.226 & 1.227 \\
\hline & 1.801 & 2.294 & 2.474 & 2.859 & 3.294 & 1.222 & 1.211 \\
\hline & 1.813 & 2.184 & 2.508 & 2.925 & 3.294 & 1.222 & 1.217 \\
\hline \multirow{3}{*}{2.0} & 1.620 & 1.899 & 2.094 & 2.375 & 3.018 & 3.052 & 1.334 \\
\hline & 1.681 & 1.798 & 2.114 & 2.372 & 3.001 & 3.507 & 1.322 \\
\hline & 1.621 & 1.895 & 2.084 & 2.385 & 3.012 & 3.506 & 1.322 \\
\hline & 1.0 & 1.2 & 1.4 & 1.6 & 1.8 & 2.0 & \\
\hline
\end{tabular}

808

(b) Difference-frequency

\begin{tabular}{|c|c|c|c|c|c|c|c|}
\hline & & 1.0 & 1.2 & 1.4 & 1.6 & 1.8 & 2.0 \\
\hline \multirow{2}{*}{1.0} & 0.666 & 0.918 & 0.982 & 1.163 & 1.347 & 1.489 & 1.575 \\
\hline & 0.668 & 0.918 & 0.982 & 1.164 & 1.347 & 1.489 & 1.575 \\
\hline \multirow{2}{*}{1.2} & 0.689 & 0.636 & 0.826 & 0.870 & 1.011 & 1.165 & 1.294 \\
\hline & 0.691 & 0.639 & 0.826 & 0.870 & 1.011 & 1.165 & 1.294 \\
\hline \multirow{2}{*}{1.4} & 0.763 & 0.640 & 0.603 & 0.772 & 0.810 & 0.925 & 1.054 \\
\hline & 0.764 & 0.643 & 0.606 & 0.772 & 0.810 & 0.925 & 1.054 \\
\hline \multirow{2}{*}{1.6} & 0.856 & 0.701 & 0.615 & 0.600 & 0.748 & 0.777 & 0.867 \\
\hline & 0.856 & 0.702 & 0.617 & 0.603 & 0.748 & 0.778 & 0.867 \\
\hline
\end{tabular}




\begin{tabular}{|c|c|c|c|c|c|c|c|}
\hline \multirow{2}{*}{1.8} & 0.943 & 0.788 & 0.678 & 0.619 & 0.615 & 0.732 & 0.749 \\
\hline & 0.941 & 0.787 & 0.678 & 0.622 & 0.618 & 0.732 & 0.749 \\
\hline \multirow{2}{*}{2.0} & 1.009 & 0.877 & 0.765 & 0.678 & 0.629 & 0.624 & 0.711 \\
\hline & 1.007 & 0.875 & 0.764 & 0.678 & 0.631 & 0.627 & 0.711 \\
\hline & 1.0 & 1.2 & 1.4 & 1.6 & 1.8 & 2.0 & \\
\hline
\end{tabular}

809 
810 Table 4 Dimensionless magnitude of the sum- and difference-frequency surge forces on

811 a vertical cylinder of $d / a=4$ for different combinations of wave headings. The upper

812 right and lower left triangle matrices contain results for the sum- and difference-

813 frequency problem respectively. The values shown are: first row, $\beta_{j}=\pi / 4$ and $\beta_{l}=0$;

814 second row, $\beta_{j}=\pi / 2$ and $\beta_{l}=0$; third row, $\beta_{j}=3 \pi / 4$ and $\beta_{l}=0$; fourth row $\beta_{j}=\pi$ 815 and $\beta_{l}=0$.

\begin{tabular}{|c|c|c|c|c|c|c|c|}
\hline$v_{a} v_{j}$ & & 1.0 & 1.2 & 1.4 & 1.6 & 1.8 & 2.0 \\
\hline \multirow{4}{*}{1.0} & 0.5282 & 1.3747 & 1.3328 & 1.0648 & 0.8620 & 0.7805 & 0.7869 \\
\hline & 0.2992 & 1.5874 & 1.9721 & 2.0936 & 1.9847 & 1.7447 & 1.4644 \\
\hline & 0.5439 & 1.5853 & 1.5824 & 1.5265 & 1.4049 & 1.2792 & 1.1684 \\
\hline & 0.6804 & 0.0000 & 3.0918 & 4.3203 & 4.3558 & 4.0296 & 3.6458 \\
\hline \multirow{4}{*}{1.2} & 0.5103 & 0.5127 & 1.7574 & 1.6591 & 1.4217 & 1.2969 & 1.2820 \\
\hline & 0.2719 & 0.3303 & 1.6146 & 1.9169 & 1.8910 & 1.6639 & 1.3720 \\
\hline & 0.4556 & 0.4166 & 1.8248 & 2.0506 & 2.1197 & 2.0459 & 1.9196 \\
\hline & 0.5448 & 0.4080 & 0.0000 & 3.5929 & 4.8941 & 4.8630 & 4.4940 \\
\hline \multirow{4}{*}{1.4} & 0.5105 & 0.4850 & 0.4748 & 2.2781 & 2.1899 & 2.0230 & 1.9493 \\
\hline & 0.2665 & 0.3027 & 0.3487 & 1.5640 & 1.6728 & 1.4821 & 1.1838 \\
\hline & 0.3996 & 0.3532 & 0.3044 & 2.0055 & 2.4706 & 2.6820 & 2.6643 \\
\hline & 0.4478 & 0.2819 & 0.1580 & 0.0000 & 4.0744 & 5.4772 & 5.4241 \\
\hline \multirow{4}{*}{1.6} & 0.5520 & 0.4907 & 0.4472 & 0.4437 & 2.8693 & 2.8363 & 2.7077 \\
\hline & 0.3108 & 0.2743 & 0.3124 & 0.3509 & 1.5547 & 1.3797 & 1.0268 \\
\hline & 0.3665 & 0.3175 & 0.2616 & 0.2067 & 2.1032 & 2.8037 & 3.1502 \\
\hline & 0.3660 & 0.1903 & 0.0676 & 0.0287 & 0.0000 & 4.5438 & 6.0609 \\
\hline \multirow{4}{*}{1.8} & 0.6202 & 0.5516 & 0.4598 & 0.4182 & 0.4269 & 3.3716 & 3.3808 \\
\hline & 0.3675 & 0.2684 & 0.2717 & 0.3067 & 0.3376 & 1.7473 & 1.3264 \\
\hline & 0.3383 & 0.2949 & 0.2393 & 0.1769 & 0.1219 & 2.1152 & 3.0308 \\
\hline & 0.2845 & 0.1296 & 0.0611 & 0.0885 & 0.1271 & 0.0000 & 5.0057 \\
\hline \multirow{4}{*}{2.0} & 0.6980 & 0.6426 & 0.5356 & 0.4333 & 0.4025 & 0.4174 & 3.6758 \\
\hline & 0.4064 & 0.2726 & 0.2431 & 0.2647 & 0.2917 & 0.3115 & 2.1971 \\
\hline & 0.3002 & 0.2680 & 0.2216 & 0.1607 & 0.0992 & 0.0494 & 2.0586 \\
\hline & 0.2337 & 0.1396 & 0.1252 & 0.1345 & 0.1421 & 0.1444 & 0.0000 \\
\hline & 1.0 & 1.2 & 1.4 & 1.6 & 1.8 & 2.0 & \\
\hline
\end{tabular}

816 
817 Table 5 Dimensionless magnitude of the sum- and difference-frequency sway forces on

818 a vertical cylinder of $d / a=4$ for different combinations of wave headings. The upper 819 right and lower left triangle matrices contain results for the sum- and difference820 frequency problem respectively. The values shown are: first row, $\beta_{j}=\pi / 4$ and $\beta_{l}=0$; 821 second row $\beta_{j}=\pi / 2$ and $\beta_{l}=0$; third row $\beta_{j}=3 \pi / 4$ and $\beta_{l}=0$.

\begin{tabular}{|c|c|c|c|c|c|c|c|}
\hline$v_{j}$ & & 1.0 & 1.2 & 1.4 & 1.6 & 1.8 & 2.0 \\
\hline \multirow{3}{*}{1.0} & 0.2478 & 0.5694 & 0.8488 & 1.2948 & 1.5325 & 1.5430 & 1.3984 \\
\hline & 0.2992 & 1.5874 & 1.1339 & 0.7558 & 0.6112 & 0.6767 & 0.8265 \\
\hline & 0.2697 & 3.8272 & 4.0536 & 4.0039 & 3.7427 & 3.3689 & 2.9771 \\
\hline \multirow{3}{*}{1.2} & 0.2421 & 0.2316 & 0.7279 & 1.2290 & 1.5836 & 1.6404 & 1.4865 \\
\hline & 0.3243 & 0.3303 & 1.6146 & 1.1564 & 0.9682 & 1.0787 & 1.2918 \\
\hline & 0.2919 & 0.2649 & 4.4055 & 4.4474 & 4.2287 & 3.8668 & 3.4607 \\
\hline \multirow{3}{*}{1.4} & 0.2717 & 0.1956 & 0.2011 & 0.9436 & 1.4655 & 1.6068 & 1.4527 \\
\hline & 0.3617 & 0.3477 & 0.3487 & 1.5640 & 1.3632 & 1.5187 & 1.8089 \\
\hline & 0.3122 & 0.2601 & 0.2601 & 4.8416 & 4.6711 & 4.3164 & 3.9081 \\
\hline \multirow{3}{*}{1.6} & 0.3388 & 0.1790 & 0.1621 & 0.1847 & 1.1885 & 1.4791 & 1.3361 \\
\hline & 0.4245 & 0.3895 & 0.3647 & 0.3509 & 1.5547 & 1.8189 & 2.2437 \\
\hline & 0.3207 & 0.2420 & 0.2328 & 0.2268 & 5.0777 & 4.7076 & 4.2565 \\
\hline \multirow{3}{*}{1.8} & 0.4170 & 0.2037 & 0.1214 & 0.1610 & 0.1896 & 1.3966 & 1.3138 \\
\hline & 0.4997 & 0.4611 & 0.4088 & 0.3661 & 0.3376 & 1.7473 & 2.4228 \\
\hline & 0.3001 & 0.2069 & 0.1929 & 0.1845 & 0.1613 & 5.1065 & 4.5666 \\
\hline \multirow{3}{*}{2.0} & 0.4863 & 0.2614 & 0.1034 & 0.1167 & 0.1754 & 0.2020 & 1.5226 \\
\hline & 0.5713 & 0.5416 & 0.4793 & 0.4052 & 0.3477 & 0.3115 & 2.1971 \\
\hline & 0.2536 & 0.1493 & 0.1381 & 0.1377 & 0.1150 & 0.0813 & 4.9699 \\
\hline & 1.0 & 1.2 & 1.4 & 1.6 & 1.8 & 2.0 & \\
\hline
\end{tabular}


823 Table 6 Dimensionless magnitude of constituent components of the sum- and 824 difference-frequency surge forces on a vertical cylinder of $d / a=4$ for $\beta_{j}=\pi / 4$ and $\beta_{j}$ $825=0$. The upper right and lower left triangle matrices contain results for the sum- and 826 difference-frequency problem respectively. The values shown are: first row, $\left|f_{j l, q, x}^{ \pm}\right|$; 827 second row, $\left|f_{j l, f, x}^{ \pm}\right|$; third row, $\left|f_{j l, b i, x}^{ \pm}\right|$.

\begin{tabular}{|c|c|c|c|c|c|c|c|}
\hline$v$ & & 1.0 & 1.2 & 1.4 & 1.6 & 1.8 & 2.0 \\
\hline \multirow{3}{*}{1.0} & 0.5282 & 1.5379 & 1.4858 & 1.4112 & 1.3369 & 1.2792 & 1.2501 \\
\hline & 0.0000 & 2.4587 & 2.4209 & 2.1482 & 1.9428 & 1.8609 & 1.8778 \\
\hline & 0.0000 & 0.4936 & 0.4521 & 0.3986 & 0.3374 & 0.2720 & 0.2068 \\
\hline \multirow{3}{*}{1.2} & 0.5305 & 0.5127 & 1.4183 & 1.3697 & 1.3217 & 1.2940 & 1.2988 \\
\hline & 0.0423 & 0.0000 & 2.8551 & 2.7754 & 2.5579 & 2.4534 & 2.4676 \\
\hline & 0.0091 & 0.0000 & 0.4164 & 0.3555 & 0.2885 & 0.2201 & 0.1564 \\
\hline \multirow{3}{*}{1.4} & 0.5262 & 0.5049 & 0.4748 & 1.4238 & 1.4008 & 1.4004 & 1.4315 \\
\hline & 0.1242 & 0.0421 & 0.0000 & 3.5218 & 3.4590 & 3.3249 & 3.2890 \\
\hline & 0.0019 & 0.0084 & 0.0000 & 0.2990 & 0.2296 & 0.1635 & 0.1130 \\
\hline \multirow{3}{*}{1.6} & 0.5228 & 0.4995 & 0.4676 & 0.4437 & 1.5435 & 1.5562 & 1.5954 \\
\hline & 0.2213 & 0.1237 & 0.0414 & 0.0000 & 4.3035 & 4.2957 & 4.2001 \\
\hline & 0.0366 & 0.0083 & 0.0074 & 0.0000 & 0.1683 & 0.1159 & 0.1027 \\
\hline \multirow{3}{*}{1.8} & 0.5230 & 0.4992 & 0.4665 & 0.4399 & 0.4269 & 1.6799 & 1.7118 \\
\hline & 0.3193 & 0.2207 & 0.1229 & 0.0406 & 0.0000 & 4.9416 & 4.9637 \\
\hline & 0.0949 & 0.0145 & 0.0108 & 0.0063 & 0.0000 & 0.1043 & 0.1357 \\
\hline \multirow{3}{*}{2.0} & 0.5260 & 0.5034 & 0.4712 & 0.4429 & 0.4253 & 0.4174 & 1.7493 \\
\hline & 0.4050 & 0.3184 & 0.2202 & 0.1213 & 0.0397 & 0.0000 & 5.2696 \\
\hline & 0.1538 & 0.0537 & 0.0020 & 0.0110 & 0.0051 & 0.0000 & 0.1872 \\
\hline & 1.0 & 1.2 & 1.4 & 1.6 & 1.8 & 2.0 & \\
\hline
\end{tabular}


829 Table 7 Dimensionless magnitude of constituent components of the sum- and 830 difference-frequency sway forces on a vertical cylinder of $d / a=4$ for $\beta_{j}=\pi / 4$ and $\beta_{l}$ $831=0$. The upper right and lower left triangle matrices contain results for the sum- and 832 difference-frequency problem respectively. The values shown are: first row, $\left|f_{j l, q, y}^{ \pm}\right|$; 833 second row, $\left|f_{j l, f, y}^{ \pm}\right|$; third row $\left|f_{j l, b i, y}^{ \pm}\right|$.

\begin{tabular}{|c|c|c|c|c|c|c|c|}
\hline$v$ & & 1.0 & 1.2 & 1.4 & 1.6 & 1.8 & 2.0 \\
\hline \multirow{3}{*}{1.0} & 0.2478 & 0.6370 & 0.5740 & 0.7194 & 0.9492 & 1.1286 & 1.2169 \\
\hline & 0.0000 & 1.0184 & 1.2788 & 1.9446 & 2.4328 & 2.6211 & 2.5600 \\
\hline & 0.0000 & 0.2045 & 0.2075 & 0.1982 & 0.1790 & 0.1523 & 0.1211 \\
\hline \multirow{3}{*}{1.2} & 0.2240 & 0.2316 & 0.5875 & 0.6995 & 0.9172 & 1.0937 & 1.1776 \\
\hline & 0.0065 & 0.0000 & 1.1826 & 1.8537 & 2.4463 & 2.6803 & 2.6082 \\
\hline & 0.0509 & 0.0000 & 0.1725 & 0.1607 & 0.1400 & 0.1133 & 0.0846 \\
\hline \multirow{3}{*}{1.4} & 0.1814 & 0.1916 & 0.2011 & 0.5898 & 0.7959 & 0.9678 & 1.0460 \\
\hline & 0.0179 & 0.0084 & 0.0000 & 1.4588 & 2.2079 & 2.5226 & 2.4455 \\
\hline & 0.1733 & 0.0397 & 0.0000 & 0.1239 & 0.1026 & 0.0778 & 0.0568 \\
\hline \multirow{3}{*}{1.6} & 0.1451 & 0.1599 & 0.1737 & 0.1847 & 0.6393 & 0.8064 & 0.8752 \\
\hline & 0.0506 & 0.0249 & 0.0107 & 0.0000 & 1.7826 & 2.2389 & 2.1630 \\
\hline & 0.3173 & 0.1371 & 0.0312 & 0.0000 & 0.0697 & 0.0514 & 0.0482 \\
\hline \multirow{3}{*}{1.8} & 0.1434 & 0.1568 & 0.1702 & 0.1815 & 0.1896 & 0.6958 & 0.7480 \\
\hline & 0.0951 & 0.0519 & 0.0293 & 0.0123 & 0.0000 & 2.0469 & 2.0114 \\
\hline & 0.4439 & 0.2535 & 0.1079 & 0.0244 & 0.0000 & 0.0432 & 0.0597 \\
\hline \multirow{3}{*}{2.0} & 0.1708 & 0.1762 & 0.1839 & 0.1914 & 0.1967 & 0.2020 & 0.7246 \\
\hline & 0.1486 & 0.0864 & 0.0515 & 0.0304 & 0.0133 & 0.0000 & 2.1827 \\
\hline & 0.5262 & 0.3551 & 0.1997 & 0.0840 & 0.0188 & 0.0000 & 0.0775 \\
\hline & 1.0 & 1.2 & 1.4 & 1.6 & 1.8 & 2.0 & \\
\hline
\end{tabular}

